\title{
Enhanced Injection Method for Synthetically Generated Turbulence within the Flow Domain of Eddy-Resolving Simulations
}

\author{
G. De Nayer, S. Schmidt, J.N. Wood, M. Breuer* \\ Professur für Strömungsmechanik, Helmut-Schmidt-Universität Hamburg, D-22043 Hamburg, Germany
}

\begin{abstract}
The quality of eddy-resolving turbulence simulations strongly depends on appropriate inflow conditions. In most cases they have to be time-dependent and satisfy certain conditions for the first (mean velocities) and second-order moments (Reynolds stresses) as well as concerning suitable length scales. To mimic a physically realistic incoming flow, synthetically generated turbulent velocity fluctuations superimposed on the mean velocity field are a valuable solution. However, the resolution of the grid near the inlet has to be sufficiently fine to avoid excessive damping of the turbulence intensity. In order to circumvent this problem, the injection of synthetically generated inflow data not at the inlet itself but inside the flow domain near the area of interest, where the grid is typically much finer, is an elegant loophole. In the present study two different injection techniques based on a source term formulation are analyzed and evaluated. In addition to these techniques the injected data are weighted by a Gaussian distribution defining the influence area. In the recent work the definition of the influence area is enhanced compared to the initial version of Schmidt and Breuer (2017) extending the application range. The case of a rather small influence area in comparison with the grid cell size is now tackled which is often relevant for industrial applications.

The flow past a wall-mounted hemisphere is chosen as test case. The bluff body is exposed to a thick turbulent boundary layer at $\mathrm{Re}=50,000$. The generation of the turbulent velocity fluctuations in the present investigation relies on the digital filter concept, but the injection techniques evaluated are not restricted to this inflow generator. The synthetic turbulent velocity fluctuations are injected about one diameter upstream of the hemisphere. Wall-resolved large-eddy simulations are carried out for two grid resolutions and the corresponding results are analyzed and compared with the reference measurements by Wood et al. (2016). Finally, one injection technique is found to be clearly superior to the other, since it guarantees the correct level of the velocity fluctuations and the reproduction of the autocorrelations.
\end{abstract}

Keywords: artificially generated turbulence, inflow generator, injection method, source term formulation, large-eddy simulation (LES), flow past hemisphere

\section{Introduction}

Large-eddy simulations and other eddy-resolving methods for the prediction of turbulent flows nowadays become essential for industrially relevant applications. Especially, LES has shown to provide a good compromise between attainable accuracy and computational effort required.

\footnotetext{
${ }^{*}$ Corresponding author

Email address: breuer@hsu-hh.de (M. Breuer)
} 
However, eddy-resolving methods are known to be more sensitive to the formulation of appropriate boundary conditions than approaches relying on the Reynolds-averaged Navier-Stokes equations $[1,4,6]$. That comprises all boundaries including rigid walls, outflows and especially inflow boundaries. Similar to the outlet the inflow boundary typically represents an artificial cut through the flow field. Consequently, LES predictions require appropriate inflow data of Dirichlet type, which adequately represent the physical flow field. For the prediction of laminar flows the specification of a steady velocity (or pressure) field at the inlet is sufficient in most cases. For turbulent flow predictions based on the Reynolds-averaged Navier-Stokes equations using for example two-equation closure models, additional specifications for the turbulence intensity and the length scales of the energy carrying vortices are sufficient.

For LES resolving partially the spectrum of turbulent length scales in the flow, appropriate boundary conditions which mimic the unsteady vortical flow at the inlet are essential [4, 25, 31]. These have to be physically meaningful instantaneous data for the entire inflow plane satisfying characteristic autocorrelations and cross-correlations of the velocity components among each other. Since the inflow boundary conditions can have a strong influence not only in the vicinity of the inlet but also on the entire flow development, the specification has to be done with reasonable care. For spatially developing flows examples can be found for DNS in e.g. $[14,16]$ and for LES in e.g. [25, 31], where in principle no differences exist between LES and DNS concerning the challenge of appropriate inflow boundary conditions.

Besides the application of periodic boundary conditions with or without rescaling techniques or the re-usage of data from auxiliary simulations, synthetically generated inflow data are a meaningful method to solve this problem. Tabor and Baba-Ahmadi [30], Fröhlich and von Terzi [9] or Sagaut et al. [20] published complete overviews of the different methods used to generate turbulent inflow data and the reader is referred to these references for more detail. Weighing all pros and cons, Schmidt and Breuer [25] choose the digital filter method of Klein et al. [14] as the basis for their extended synthetic turbulence inflow generator (STIG) for hybrid LES-URANS applications. This method allows to generate inflow data with low computational effort only necessitating time-averaged velocities, Reynolds stresses and three integral length scales. These required data can be easily provided by experiments or direct numerical simulations, making the digital filter method of Klein et al. [14] popular. Schmidt and Breuer [25] evaluated the digital filter method with success based on two classical wall-bounded internal flows. i.e., the plane channel flow [17] and the flow over periodic hills [7].

During the application of the synthetic turbulence inflow generator to more challenging test cases, a critical issue was noticed, which is general and not restricted to the digital filter method. At or close to the inflow region the grids generated for the prediction of external flows around obstacles such as airfoils often possess a relatively coarse resolution resulting from the grid stretching from the obstacle towards the outer boundaries. Consequently, a LES prediction can not resolve the small flow structures in this region leading to a strong damping effect of the synthetic turbulent inflow fluctuations. A solution to this problem is to inject the fluctuations closer to the region of interest, where the grid is fine enough. Schmidt and Breuer [27] suggested a first injection method (denoted STIG-D in the present work): The artificial turbulence is superimposed to the fluid flow at a plane inside the domain based on a source-term formulation relying on a temporal derivative. The technique is also combined with a Gaussian bell-shape distribution of the source term in space in order to mimic a 3D influence area and to augment the propagation of the synthetic fluctuations in streamwise direction. The procedure was evaluated based on the flow past a SD7003 airfoil at Re $=$ 60,000 . In this application the integral length scale in streamwise direction is much larger than the cell size at the injection area. Thus, the width of the Gaussian function can be set 
Computers \& Mathematics with Applications, vol. 75 (7), pp. 2338-2355, (2018).

according to this integral scale. The STIG-D method was also applied in the LES prediction of the flow around a wall-mounted hemisphere by Wood et al. [31]. Here the integral length scale in streamwise direction was found to be smaller than the grid resolution. Hence, in order to artificially correct the turbulent inflow conditions, the width of the Gaussian distribution was ad-hoc adjusted to a value deviating from the streamwise integral length scale. For this purpose, several LES predictions were required which results in additional high computational costs. A second problem related to the STIG-D injection method was found by Schmidt and Breuer [26], i.e., it does not preserve the autocorrelations of the fluid flow.

In order to avoid the deviations of the autocorrelations, an enhanced injection method (denoted STIG-R in the present work) was developed based on a source term formulation which relies on a relaxation method. The spatial Gaussian distribution is used again to form the threedimensional influence area. Schmidt and Breuer [26] validated this new injection technique based on the airfoil flow using a grid which is relatively fine compared to the integral length scale at the injection region. Since that is not the case for all applications, the injection technique has to be extended to support a large range of the integral length scales for a given grid resolution without empirical adjustments. That is the central objective of the present work including a thorough evaluation of the extended method based on the wall-mounted hemisphere flow [31].

\section{Injection method for synthetic turbulence within the domain}

As discussed above the application of appropriate temporally and spatially correlated velocity distributions as inlet boundary conditions is essential to predict realistic flow fields. For this purpose, the digital filter concept of Klein et al. [14] is applied as a synthetic turbulence inflow generator (STIG) in the present study. It provides instantaneous three-dimensional velocities relying solely on the definition of one integral time scale $T$, two integral length scales ${ }^{1}$ (e.g., $L_{y}$ and $L_{z}$ ) and the distributions of the mean velocity and Reynolds stresses.

\subsection{Length scales, integral length scales and turbulent structures of the synthetic turbulence}

The integral time scale $\mathscr{T}$ as well as the integral length scale $\Lambda$ rely per definition either on the autocorrelation or the two-point correlations in the two directions within the inflow plane, respectively [25]. In the digital filter concept of Klein et al. [14], the autocorrelation function $R_{\text {uu }}$ is described by a Gaussian bell shape assuming homogeneous turbulence in a late stage. This special shape implies that the length scale $L$ used in the definition of $R_{\text {uu }}$ is equal to the integral length scale $\Lambda$ of the problem:

$$
\Lambda=\int_{0}^{\infty} R_{\mathrm{uu}}(r) d r=\int_{0}^{\infty} \exp \left(-\frac{\pi r^{2}}{4 L^{2}}\right) d r=\sqrt{\frac{\pi}{\frac{\pi}{4 L^{2}}}} / 2=L .
$$

The integral length scale is related to the size of the turbulent structures, but not equal to its extension. The largest turbulent structure, also called macrostructure, is approximately delimited by the two-point correlations tending towards zero. Therefore, the largest structure is always larger than the value of the integral length scale for each direction. The method of Klein et al. [14] is based on digital filters. Thus, all the generated structures have a size of the same order of magnitude. In Klein et al. [14], the half filter width $W / 2$ is set to twice the

\footnotetext{
${ }^{1}$ Note that in the present study equal length scales are assumed in the different directions, i.e., $L_{y}=L_{z}=L$.
} 
Computers \& Mathematics with Applications, vol. 75 (7), pp. 2338-2355, (2018).

integral length scale $L$. Therefore, the size of the largest turbulent structure present in the synthetic data is about four times the integral length scale $L$.

In order to take these synthetically generated turbulent structures in the simulation into account, the grid resolution has to be adjusted accordingly. If the cell size is larger than these structures, they can not be resolved.

\subsection{Source term formulation}

At the inflow region the grid has typically a coarse resolution resulting from the grid stretching from the inner to the outer domain. Thus, small flow structures can not be resolved. Combined with hardly avoidable damping effects of the numerical scheme on coarse grids, the synthetically generated turbulence is typically hindered to reach the region of interest. In order to circumvent this problem, a shift towards finer resolved areas within the computational domain is meaningful. This methodology requires the injection of the artificial turbulence as a source term inside the domain. In general, the idea to use a source term formulation for this purpose is not new and has been used before by others, e.g., [12, 29].

To formulate a source term injection method, Schmidt and Breuer [26] considered at first the integral form of a general momentum conservation equation for a general quantity $\phi(\phi \hat{=}$ $u, v$, and $w)$ as used in the finite-volume scheme:

$$
\int_{\mathrm{V}} \frac{\partial(\rho \phi)}{\partial t} d V+\int_{\mathrm{S}}\left(\rho u_{\mathrm{j}} \phi-\Gamma_{\phi} \frac{\partial \phi}{\partial x_{\mathrm{j}}}\right) \cdot n_{\mathrm{j}} d S=S_{\phi} .
$$

$\Gamma_{\phi}$ represents the diffusion coefficient and $S_{\phi}$ the source terms not depending on the STIG. $V$ is the volume of the cell and $S$ its surface. $t$ denotes the physical time. In order to inject the artificial turbulence generated by the STIG, a supplementary source term $S_{\phi}^{\text {syn }}$ is added. As a consequence, the flow field is altered and denoted $\phi^{*}$. Assuming that the usual source term $S_{\phi}$, the convective and diffusive fluxes are not immediately influenced, Equation (2) now reads:

$$
\int_{\mathrm{V}} \frac{\partial\left(\rho \phi^{*}\right)}{\partial t} d V+\int_{\mathrm{S}}\left(\rho u_{\mathrm{j}} \phi-\Gamma_{\phi} \frac{\partial \phi}{\partial x_{\mathrm{j}}}\right) \cdot n_{\mathrm{j}} d S=S_{\phi}+S_{\phi}^{\mathrm{syn}} .
$$

The formulation of the STIG source term is obtained by subtracting Eq. (2) from Eq. (3):

$$
S_{\phi}^{\mathrm{syn}}=\int_{\mathrm{V}} \frac{\partial\left(\rho \phi^{*}\right)}{\partial t} d V-\int_{\mathrm{V}} \frac{\partial(\rho \phi)}{\partial t} d V=\int_{\mathrm{V}} \frac{\partial\left(\rho \phi^{\prime}\right)}{\partial t} d V,
$$

where $\phi^{\prime}$ is the difference between the altered and the original quantity and describes the velocity fluctuations.

In the first source-term method, denoted STIG-D, Schmidt and Breuer [26] set the fluctuation value $\phi^{\prime}$ to the synthetically generated fluctuation value $\left(\phi^{\prime}\right)^{\text {syn }}$, which leads to:

$$
\phi^{*}=\phi+\left(\phi^{\prime}\right)^{\mathrm{syn}} .
$$

Therefore, the STIG-D source-term results in:

$$
\left.S_{\phi}^{\mathrm{syn}}\right|_{S T I G-D}=\int_{\mathrm{V}} \frac{\partial\left(\rho\left(\phi^{\prime}\right)^{\mathrm{syn}}\right)}{\partial t} d V .
$$

The STIG-D source term is directly based on the temporal derivative (STIG-D: D for derivative) of $\left(\phi^{\prime}\right)^{\text {syn }}$. The first derivative in time is approximated by a backward differencing scheme:

$$
\left.S_{\phi}^{\mathrm{syn}}\right|_{S T I G-D} \approx \int_{\mathrm{V}} \rho \frac{\left(\phi^{\prime}\right)_{t+\Delta t}^{\mathrm{syn}}-\left(\phi^{\prime}\right)_{t}^{\mathrm{syn}}}{\Delta t} d V
$$


Applying the STIG-D source term for a simulation of a channel flow, a thorough analysis of the autocorrelations [26] showed significant deviations from the theoretical autocorrelations defined by the predefined scales. By analyzing the STIG-D method, it was found that the starting assumption $\phi^{*}=\phi+\left(\phi^{\prime}\right)^{\text {syn }}$ is responsible for these observations. It means that the injected perturbation $\left(\phi^{\prime}\right)^{\text {syn }}$ is assumed to immediately modify the variable $\phi$ to the altered value $\phi^{*}$. In reality an injected perturbation influences the variable with a certain delay given by the relaxation time. Therefore, in order to ensure the correct physical representation of the autocorrelations, Schmidt and Breuer searched for an enhanced formulation of $S_{\phi}^{\text {syn }}$. They assumed that the previously mentioned relaxation time is related to the integral time scale $T$ (STIG-R: R for relaxation). This leads to the starting assumption for the enhanced sourceterm method STIG-R:

$$
\phi^{*}=\phi+\frac{\Delta t}{T}\left(\phi^{\prime}\right)^{\mathrm{syn}}
$$

By substituting $\phi^{*}$ in Eq. (4) the STIG-R source term is expressed as:

$$
\begin{aligned}
\left.S_{\phi}^{\mathrm{syn}}\right|_{\text {STIG-R }} & =\int_{\mathrm{V}} \frac{\partial\left(\rho \phi^{*}\right)}{\partial t} d V-\int_{\mathrm{V}} \frac{\partial(\rho \phi)}{\partial t} d V \\
& =\int_{\mathrm{V}} \frac{\partial\left(\rho \phi+\rho \frac{\Delta t}{T}\left(\phi^{\prime}\right)^{\mathrm{syn}}\right)}{\partial t} d V-\int_{\mathrm{V}} \frac{\partial(\rho \phi)}{\partial t} d V \\
& =\int_{\mathrm{V}} \rho \frac{\Delta t}{T} \frac{\partial\left(\left(\phi^{\prime}\right)^{\mathrm{syn}}\right)}{\partial t} d V .
\end{aligned}
$$

As for STIG-D the first derivative in time is approximated by a backward differencing scheme. However, contrary to STIG-D, a second assumption is made [26]: For STIG-R the flow at the time step $t$ has the correct autocorrelation and is undisturbed. It means that $\phi_{t}^{*}=\phi_{t}$ or $\left(\phi^{\prime}\right)_{t}^{\text {syn }}=0$. Thus, it follows:

$$
\begin{aligned}
\left.S_{\phi}^{\mathrm{syn}}\right|_{S T I G-R} & \approx \int_{\mathrm{V}} \rho \frac{\Delta t}{T} \frac{\left(\phi^{\prime}\right)_{t+\Delta t}^{\mathrm{syn}}-\left(\phi^{\prime}\right)_{t}^{\mathrm{syn}}}{\Delta t} d V \\
& \approx \int_{\mathrm{V}} \rho \frac{\left(\phi^{\prime}\right)_{t+\Delta t}^{\mathrm{syn}}}{T} d V .
\end{aligned}
$$

To summarize, instead of using the temporal derivative of the synthetic velocity fluctuations as done in STIG-D, in STIG-R the synthetic velocity fluctuations at the computed time step are now directly scaled by the inverse of the integral time scale $T$. This characteristic time scale can be physically interpreted as a relaxation time describing a certain time interval required by the system to react to the introduced velocity fluctuations. It is important to stress that the relaxation time is not chosen arbitrarily but given by the integral time scale $T$. Thus, this source term formulation does not demand any additional empirical parameter. Instead, the required parameter is directly coupled to the synthetic inflow generator. Note that the choice of the required integral time scale $T$ as well as the integral length scale $L$ is explained in Section 3.2. With regard to the synthetic turbulence inflow generator $L$ and $T$ describe the size of the characteristic scales found in the generated inflow data. The revised method was shown to guarantee the correct distribution of the autocorrelations [26].

\subsection{Assurance of an adequate influence area}

The synthetic turbulence data are generated by the STIG in a 2D plane. This is suited for the case when the data are applied at the inflow. However, in order to inject the data in a 3D 
domain guaranteeing the correct integral length scale in the streamwise direction, an additional treatment based on a Gaussian bell-shape weighting is applied. The Gaussian distribution is chosen since it fits to the definition of the filter coefficients by Klein et al. [14]. It forms a $3 D$ influence area with the dimension $L_{\text {inf }}$ equal to twice the integral length scale $L$ (in the main flow direction). Two scenarios exist:

- The first is sketched in Fig. 1. The grid is fine in comparison to $2 L$. In other words, several control volumes (at least three) exist in the influence area defined by $L_{\mathrm{inf}}=2 L$. In this case the source terms are weighted in the vicinity of the injection plane according to:

$$
S_{\phi}^{\mathrm{CFD}}\left(x_{\mathrm{i}}\right)=S_{\phi}^{\mathrm{syn}} \exp \left(-\frac{\pi}{2} \frac{\left(\Delta d_{\mathrm{i}}\right)^{2}}{L^{2}}\right)
$$

where $\Delta d_{\mathrm{i}}$ is defined as the distance in the i-direction between the origin of the influence area (injection plane) and the current position $x_{\mathrm{i}}$.

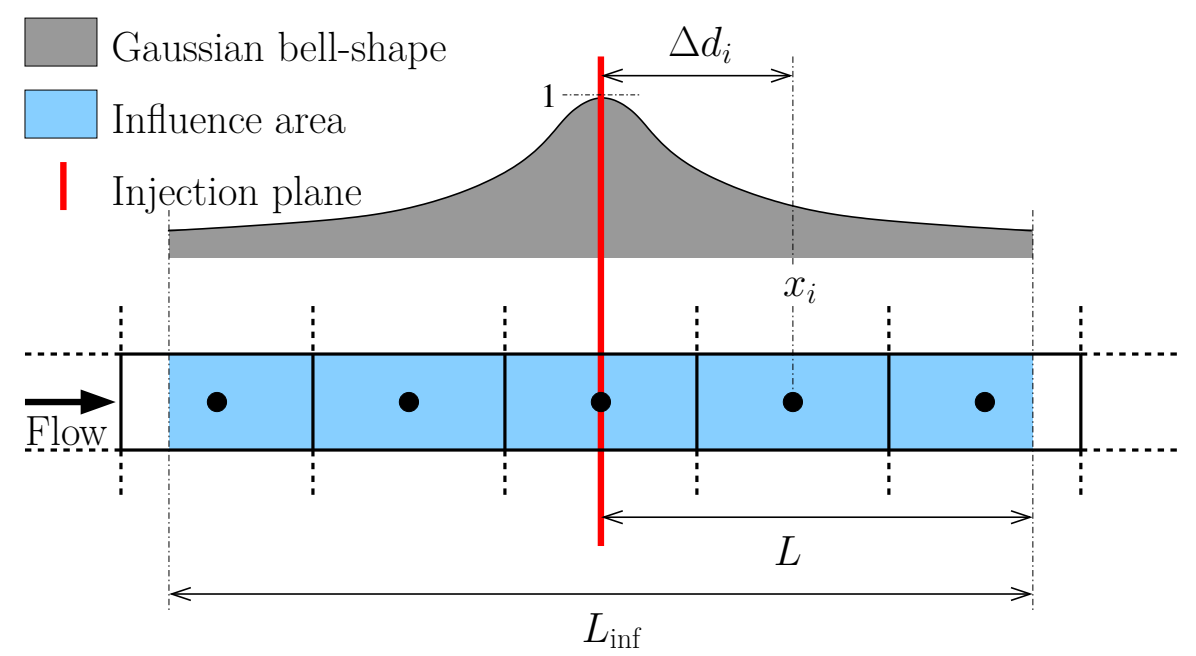

Figure 1: First case: Fine grid resolution in comparison to $L_{\text {inf }}=2 L$ : Application of a Gaussian bell-shape distribution of the source term to mimic a 3D influence area.

- The second case is sketched in Fig. 2. Here, the influence area $L_{\text {inf }}=2 L$ is smaller than the distance between the cell centers of the two neighboring control volumes (i.e., $\left.L_{\text {inf }}<2 \Delta d_{0}\right)$. In this case the effect of the Gaussian bell-shape source term distribution can solely be applied on this central cell. For this purpose, a correction factor based on $L$ and $\Delta d_{0}$ is required. In order to evaluate the global effect of the Gaussian bell-shape weighting, it is integrated over the entire influence area:

$$
\int_{-L}^{L} \exp \left(-\frac{\pi}{2}\left(\frac{x}{L}\right)^{2}\right) d x=\sqrt{2} \operatorname{erf}\left(\sqrt{\frac{\pi}{2}}\right) L .
$$

Thus, in this case the source term solely applied at the injection plane has to be scaled to fit the cell size $\Delta d_{0}$ as follows:

$$
S_{\phi}^{\mathrm{CFD}}\left(x_{\mathrm{i}}\right)=S_{\phi}^{\mathrm{syn}} \sqrt{2} \operatorname{erf}\left(\sqrt{\frac{\pi}{2}}\right) \frac{L}{\Delta d_{0}} \approx S_{\phi}^{\mathrm{syn}} 1.3063 \frac{L}{\Delta d_{0}} .
$$




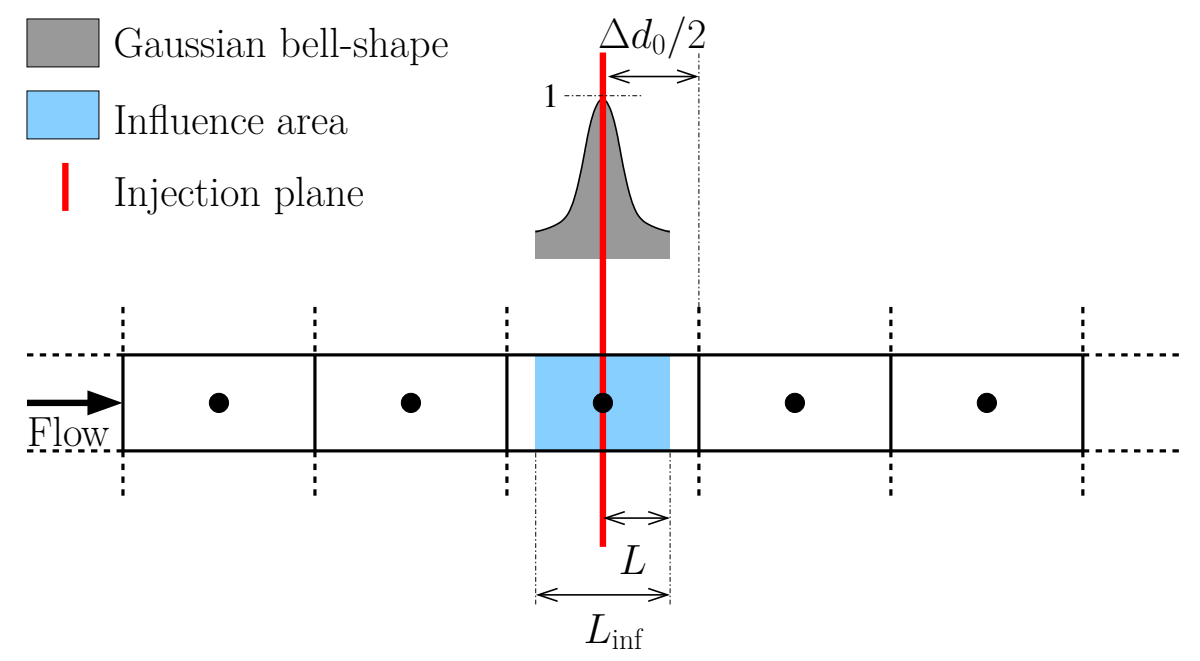

Figure 2: Second case: Coarse grid resolution in comparison to $L_{\text {inf }}=2 L$ : Application of a data scaling.

\subsection{Validation of the method}

As mentioned above the initial source-term method STIG-D was evaluated based on the flow around an airfoil [26] and based on the flow around a hemispherical obstacle [31]. In the latter the integral length scale in streamwise direction required for the STIG is smaller than the cell size. Since that was found to lead to a strong decrease of the imposed velocity fluctuations, the Gaussian weighting function according to Eq. (9) was empirically adjusted to assure the correct turbulence intensity at the injection plane. That is an obvious drawback of STIG-D. The second critical issue found by Schmidt and Breuer [26] was that STIG-D does not guarantee correct autocorrelations. That was solved by the development of the enhanced source-term injection method STIG-R. Based on the flow around the airfoil they successfully validated the enhanced method and showed that it is superior to STIG-D. In their test case the integral length scale in streamwise direction was much larger than the cell size. Consequently, the 3D influence area is defined according to the first scenario in Section 2.3.

In order to finalize the validation of the method, the enhanced STIG-R method combined with the 3D influence area presented in Section 2.3 for a coarse grid resolution in comparison to $L_{\text {inf }}$ (second scenario) has to be thoroughly studied. That is the objective of the present work, which relies again on the hemisphere flow.

\section{Description of the test case}

In environmental and civil engineering surface-mounted hemispherical bluff bodies are commonly used as architectural design elements (see Fig. 3). Although the hemisphere represents a simple geometry, it exhibits a rather complicated flow field including complex flow patterns. They can be roughly classified into an upstream horseshoe vortex system and a recirculation area with trailing vortices in the wake region. If these bluff bodies are exposed to turbulent boundary layers, the complexity of the flow field increases significantly. Therefore, Wood et al. [31] conducted complementary experimental and numerical investigations of the turbulent flow around a wall-mounted hemispherical obstacle relying on the STIG-D procedure with the rather crude ad-hoc modifications mentioned above. The experimental reference data available on the ERCOFTAC Knowledge Base Wiki in the category 'Semi-confined Flows' accessible as case 3-33 under http://qnet-ercoftac.cfms.org.uk/w/index.php/UFR_3-33 are used for 
the evaluation of the new simulations based on STIG-R and STIG-D combined with the adequate 3D influence area. It has to be noted that the STIG-D results below differ from the STIG-D data published in [31]. In the current work the width of the 3D influence area $L_{\text {inf }}$ is set to twice the integral length scale $L$ (following scenario 2 of Section 2.3), whereas in [31] it was empirically adjusted to give the correct turbulence level after the injection plane.

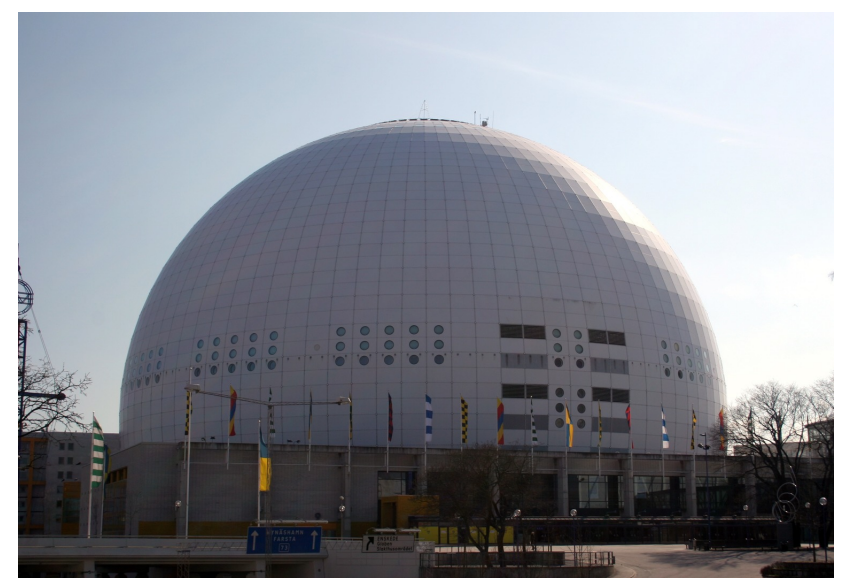

(a) Ericsson Globe in Stockholm (by Tage Olsin).

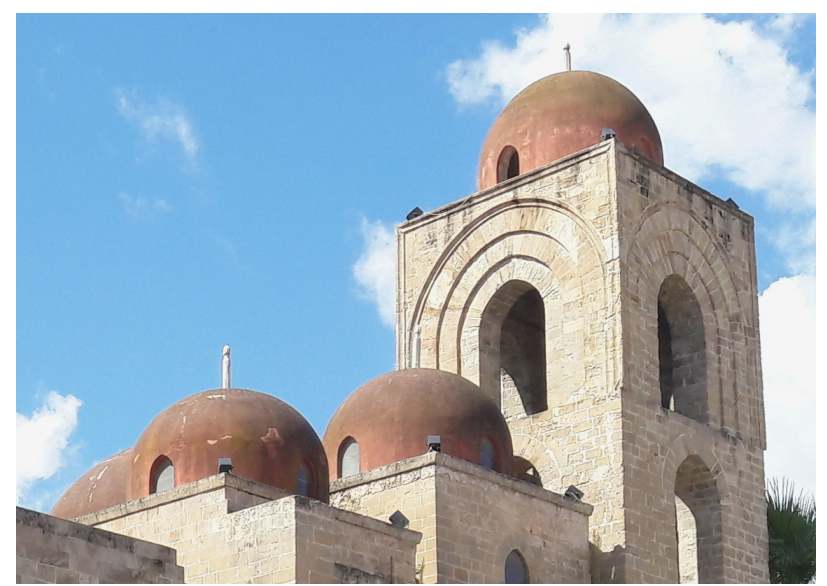

(b) San Giovanni degli Eremiti in Palermo (by Madlen Jahncke).

Figure 3: Examples of hemispherical domes in civil engineering.

\subsection{Geometry and physical properties}

The investigated hemispherical body (diameter $D$ ) is mounted on an uniform smooth wall as depicted in Fig. 4. The structure is put into a thick turbulent boundary layer, where the distribution of the time-averaged velocity in main flow direction closely follows the $1 / 7$ power law. At a distance of 1.5 diameters upstream of the bluff body the thickness of the boundary layer $\delta$ corresponds to the height of the hemisphere $(\delta=D / 2)$. The characteristics of the boundary layer were determined based on laser-Doppler anemometry (LDA) and constanttemperature anemometry (CTA) in a wind tunnel with an open test section [31]. As visible in Fig. 5 besides the mean velocity all relevant Reynolds stresses were determined as a function of the wall distance. These data are the basis for the generation of the inflow data for the present predictions. The Reynolds number of the air flow $\left(\rho=\rho_{\text {air }}=1.225 \mathrm{~kg} / \mathrm{m}^{3}, \mu_{\text {air }}=\right.$ $18.27 \times 10^{-6} \mathrm{~kg} /(\mathrm{m} \mathrm{s})$ at $\left.\vartheta=20^{\circ} \mathrm{C}\right)$ is set to $\mathrm{Re}=\rho_{\text {air }} D U_{\infty} / \mu_{\text {air }}=50,000 . U_{\infty}$ is the undisturbed free-stream mean velocity in $x$-direction at standard atmospheric conditions. The Mach number is low $(\mathrm{Ma} \leq 0.03)$. The air flow can be assumed to be incompressible and isotherm.

The results will be given in dimensionless form based on $D, U_{\infty}$ and $\rho_{\text {air }}$. The origin of the frame of reference is taken at the center of the base area of the hemisphere, where $x$ denotes the streamwise, $y$ the spanwise and $z$ the vertical (wall-normal) direction.

\subsection{Numerical methodology and setup}

To predict the turbulent flow based on the large-eddy simulation technique, an enhanced version of the 3D finite-volume fluid solver FASTEST-3D is used [5]. The discretization is based on a curvilinear, block-structured body-fitted grid with a colocated variable arrangement. The technique of flux blending [8] is applied for the spatial discretization of the convective fluxes to stabilize the simulation (solely $5 \%$ first-order accurate upwind scheme combined with $95 \%$ second-order accurate central scheme as in [31]). The temporal discretization is of 


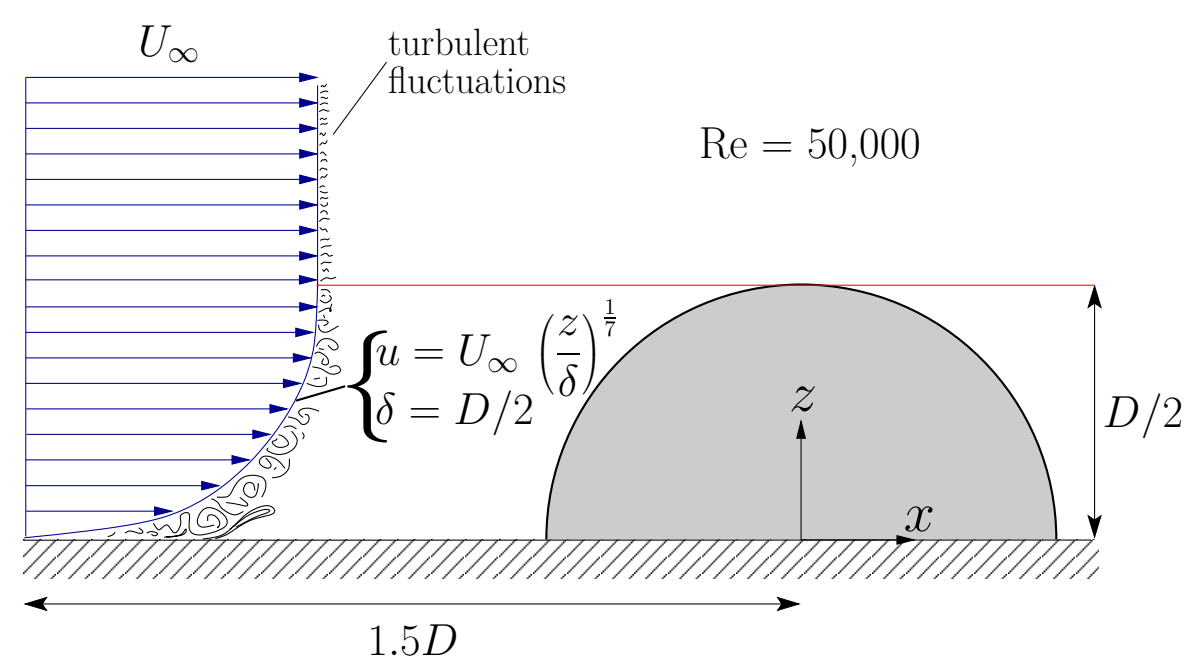

Figure 4: Surface-mounted hemisphere within a turbulent boundary layer [31].

second-order accuracy relying on a classical predictor-corrector scheme. The solver is efficiently parallelized based on the domain decomposition technique relying on non-blocking MessagePassing-Interface (MPI) communications [23].

Since LES is used, the large scales of the turbulent flow field are resolved directly, whereas the non-resolvable small scales have to be taken into account by a subgrid-scale (SGS) model. Owing to the moderate Reynolds number considered and the fine grid applied, the SGS model is expected to have a limited influence on the results. A preliminary study on the effect of the SGS model [31] for the classical Smagorinsky [28] combined with the Van-Driest damping function, the dynamic Smagorinsky [11] and the WALE model [18] demonstrated that the Smagorinsky model is appropriate for this case. Thus, it is used here applying the standard parameter $C_{s}=0.1$ and Van-Driest damping.

To simulate the flow using a block-structured mesh, the chosen computational domain is a large hemispherical expansion (radius $10 D$ ) with its origin at the center of the hemisphere. The final high-quality grid contains $30.72 \times 10^{6}$ control volumes $(\mathrm{CVs})$ and is denoted reference grid. The maxima of the time-averaged $z^{+}$values (wall-normal direction) on the wall are below 0.25 . The aspect ratio of the cells on the hemispherical body are low. This yields a dimensionless cell size in the two tangential directions below 29, which fits to the classical recommendation for a wall-resolved LES by Piomelli and Chasnov [19]. For this fine grid a small time step of $\Delta t^{*}=\Delta t U_{\infty} / D=3.084 \times 10^{-5}$ is required ensuring a CFL-number below unity. To investigate the effect of the grid resolution, the grid is coarsened leading to a medium grid composed of $4.3 \times 10^{6} \mathrm{CVs}$. Similar to the reference grid the viscous sublayer is fully resolved and the first cell center is approximately located at a distance of $\Delta z / D \approx 5 \times 10^{-5}$ from the wall. The aspect ratio of the cells on the hemispherical body remains nearly the same as on the fine grid and thus is in the range between 1 and 10. The geometric stretching ratios are slightly increased compared to the reference grid but kept below 1.1. Based on these parameters the dimensionless cell sizes in the wall-normal and tangential directions still fit to the recommendations [19] for wall-resolved LES. Due to the grid coarsening the LES time step can be increased to $\Delta t^{*}=\Delta t U_{\infty} / D=1.028 \times 10^{-4}$.

Since the predictions on both grids are a wall-resolved LES, the bottom of the domain and the surface of the hemisphere are no-slip walls. The outer surface of the hemispherical expansion is divided into an inlet and outlet patches. At the outlet a combination of a convective boundary 


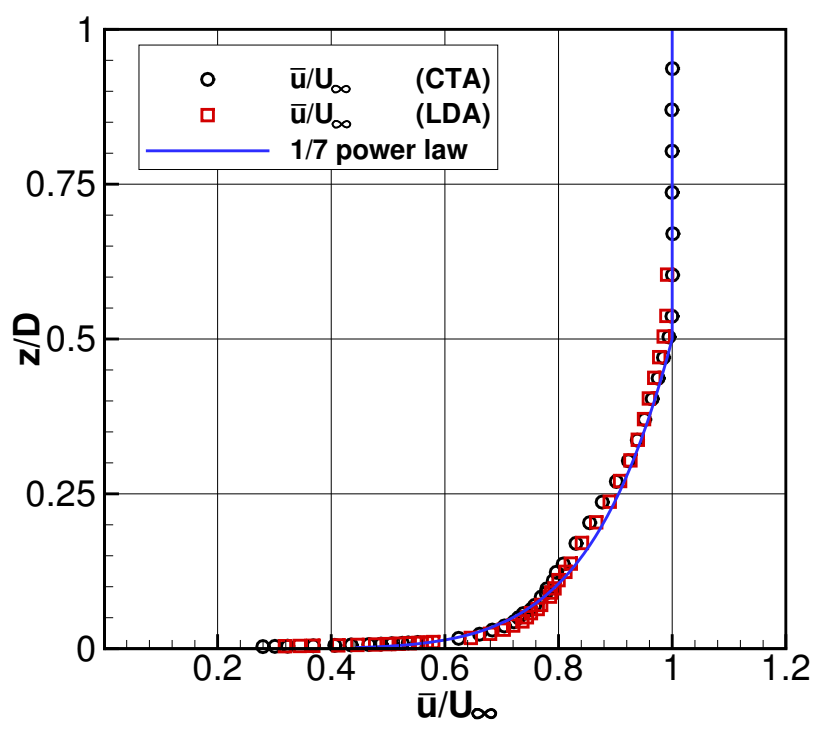

(a) Streamwise mean velocity profile.

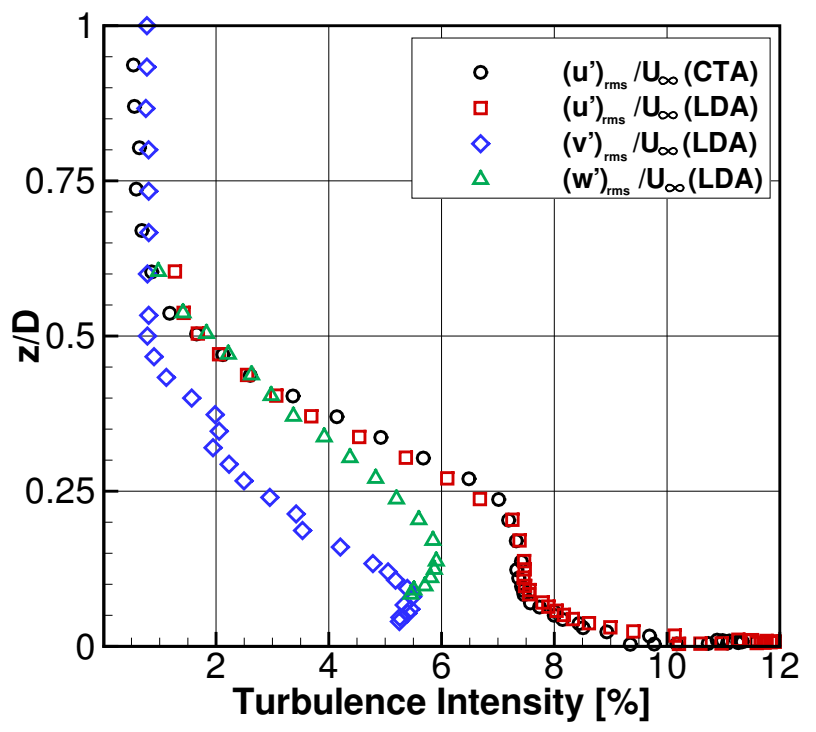

(c) $\left(u^{\prime}\right)_{r m s} / U_{\infty},\left(v^{\prime}\right)_{r m s} / U_{\infty}$ and $\left(w^{\prime}\right)_{r m s} / U_{\infty}$.

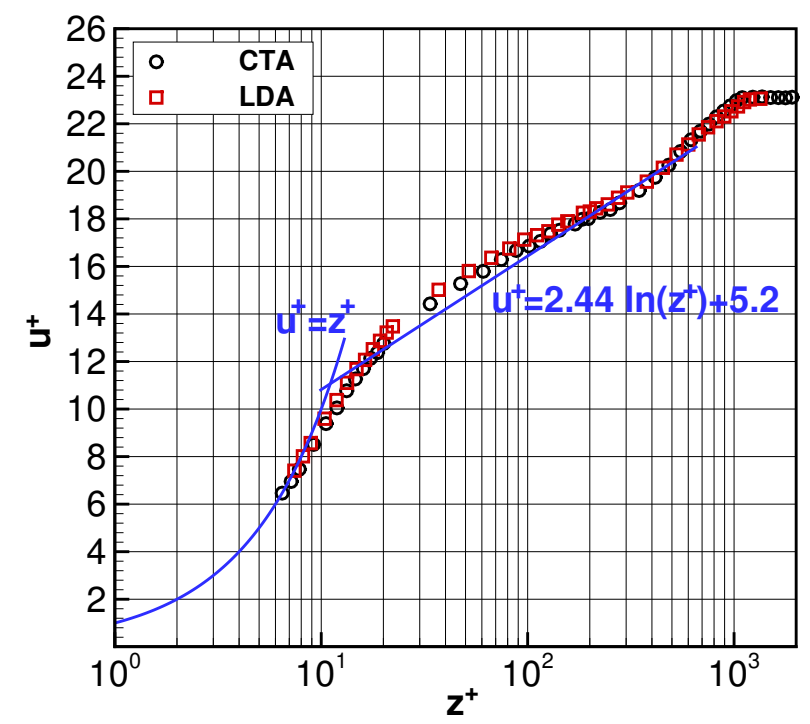

(b) $z^{+}-u^{+}$distribution.

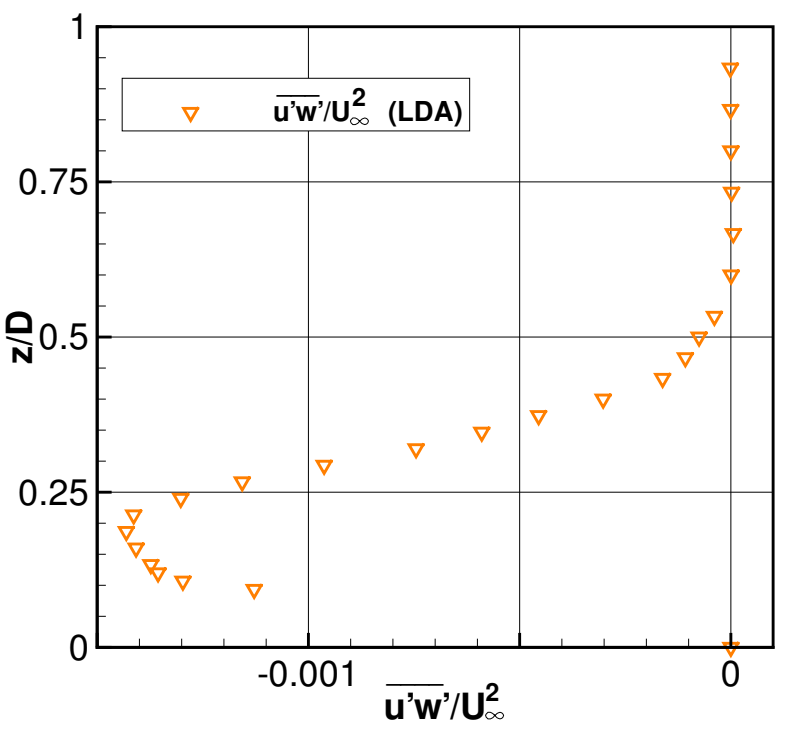

(d) $\overline{u^{\prime} w^{\prime}} / U_{\infty}^{2}$.

Figure 5: Inflow properties of the turbulent boundary layer at the inlet of the test section $(x / D=-1.5)$.

condition and a zero velocity gradient boundary is applied. The latter is used in those regions not strongly influenced by vortical flow structures. The convective velocity is set according to the $1 / 7$ power law. For further details about the boundary conditions, please refer to Wood et al. [31].

For the generation of the synthetic inflow data the time-averaged velocities and Reynolds stresses measured in the experiment (see Fig. 5) are taken. Since it was not possible to determine all Reynolds stress components in the direct vicinity of the wall, these measurements have been complemented by DNS data of Schlatter et al. [24]. Furthermore, the open question remains, how to set appropriate time and length scales. Although these quantities typically vary in a turbulent boundary layer as a function of the wall distance, the digital filter concept of Klein et al. [14] solely allows to define a constant value within the entire boundary layer. Thus, a reasonable compromise is required. In the present case, only the turbulent structures injected at $z / D<0.5\left(z^{+}<1140\right)$ will impact on the bluff body and therefore will mainly drive the flow around the hemisphere. Moreover, looking at the distribution of the turbulence 
intensity (see Fig. 5(c)), the most intensive structures will be at $z / D \lesssim 0.3\left(z^{+} \lesssim 680\right)$. The dominant structures present in a turbulent boundary layer are the streaks and the hairpin vortices. Since the streaks are confined near the wall (between $5<z^{+}<50$ in the buffer layer), it was decided in Wood et al. [31] to generate synthetic turbulent structures of the size of typical hairpin vortices. The hairpin structures have a characteristic streamwise dimension of $\Delta x^{+}=200$ [3] or 300 [15] near the wall and of $\Delta x^{+}=140$ in the logarithmic region, a spanwise extension of about $\Delta y^{+}=100$ [2] and a height between $\Delta z^{+}=200$ and 400 [2].

Owing to the different length scales a reasonable compromise has to be found. Using the classical length scale specification for the $\log$-law region $L=\kappa z$ applied in the mixing length concept by Prandtl, the integral length scale in wall-normal direction is computed at $z^{+}=100$ with $\kappa=0.41$. It leads to $L / D=2.06 \times 10^{-2}$. This value is also applied for the spanwise and streamwise integral length scale producing synthetic turbulent structures with a dimensionless size of about $\Delta x^{+}=\Delta y^{+}=\Delta z^{+}=4 L \rho u_{\tau} / \mu \approx 185$ in each direction (see Section 2.1). This is a good compromise between the size of the hairpin structures in streamwise, spanwise and wall-normal direction. The dimensionless integral time scale is deduced with the help of the Taylor hypothesis and set to $T U_{\infty} / D=L U_{\infty} /\left(D u\left(z^{+}=100\right)\right)=2.79 \times 10^{-2}$.

Moreover, the size of these macrostructures are larger than or equal to the cell size of the reference $\left(\Delta x^{+}=75\right)$ and medium grids $\left(\Delta x^{+}=180\right)$, respectively. As already mentioned in Section 2.1, a too small value of the integral length scale leads to small structures which can not be resolved. Therefore, a compromise has to be found between the integral length scale and the grid resolution.

For both STIG-D and STIG-R methods, the 3D influence area is defined by one of the two Gaussian distributions described in Section 2.3. In the present setup the integral length scale in streamwise direction $L$ is rather small. Even on the reference grid $L_{\text {inf }}=2 L$ is slightly shorter than twice the cell size of the injection plane. Therefore, the source term has to be scaled according to the second scenario given by Eq. (11). In the present case $L_{\text {inf }} / D=4.12 \times 10^{-2}$, whereas $\Delta d_{0} / D=3.33 \times 10^{-2}$ and $8.0 \times 10^{-2}$ for the reference and the medium grid, respectively. It leads to $S_{\phi}^{\mathrm{CFD}}\left(x_{\mathrm{i}}\right) \approx 0.81 S_{\phi}^{\text {syn }}$ for the reference grid and to $S_{\phi}^{\mathrm{CFD}}\left(x_{\mathrm{i}}\right) \approx 0.33 S_{\phi}^{\text {syn }}$ for the medium grid.

For both methods the synthetically generated data are limited to 480,000 time steps (corresponding to about 15 dimensionless time units), so that the same inflow data have to be reused several times during the whole simulation. In order to store the entire time series about 160 GBytes are for example required for the reference grid. The values are reasonable since only two-dimensional planes of the inflow data have to be stored. Furthermore, compared to the main LES predictions the CPU-time requirements for the generation of the inflow data are marginal (only about $0.33 \%$ for the reference grid). This is due to the fact that the original formulation of Klein et al. [14] was replaced by the much more efficient procedure suggested by Kempf et al. [13] surrogating the cubic dependence of the computational effort from the support of the filter by a linear relation.

\section{Results and discussion}

The unsteady flow patterns past a hemisphere can be classified into several regions. These regions are named based on the terminology used by Savory and Toy in their experiments investigating the effect of different turbulent boundary layers approaching a wall-mounted hemisphere [21, 22]. Figure 6 exemplarily highlights these regions based on the instantaneous pressure distribution and vorticity magnitude in the symmetry plane at an arbitrarily chosen instant in time. The hemispherical bluff body acts as an obstacle which leads to a positive 
pressure gradient in front of the hemisphere so that the boundary layer separates from the ground forming the horseshoe vortex system (1). Upstream of the hemisphere this horseshoe vortex system dominates. The stagnation area on the surface of the hemisphere is located at about 15 degrees above the ground plate (2). The flow is accelerated along the contour of the hemisphere (3). At about the apex of the hemisphere the flow detaches (4). As a consequence of the flow separation a shear layer (5) and a recirculation area appear. The latter is separated from the outer field by a dividing streamline. Strong shear layer vorticity can be observed leading to the generation of Kelvin-Helmholtz vortices (6) which travel downstream in the flow field. In the reattachment region (7) the flow impinges on the wall and a splatting effect occurs, redistributing momentum from the wall-normal direction to the streamwise and spanwise directions.

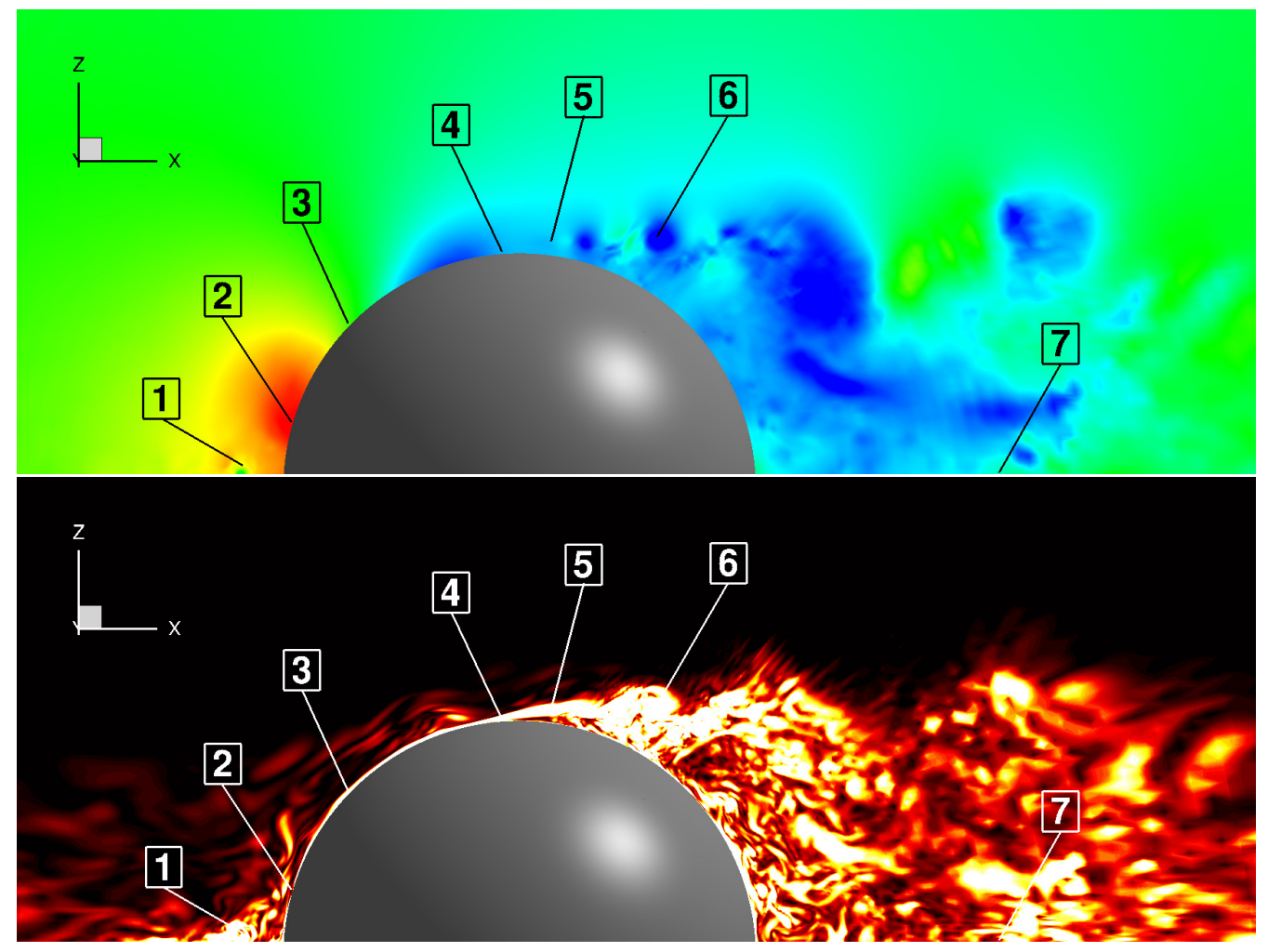

Figure 6: Snapshot of the instantaneous flow in the symmetry plane of the hemisphere: Visualization of flow regions and characteristic flow features: (1) horseshoe vortex system, (2) stagnation point, (3) acceleration of the flow, (4) separation point, (5) dividing streamline, (6) shear layer vorticity, (7) reattachment point. (Top: Pressure; Bottom: Vorticity magnitude).

\subsection{Evaluation of the two injection techniques $S T I G-D$ and $S T I G-R$}

To evaluate the performance of the STIG-D and STIG-R methods described in Section 2, two wall-resolved LES are carried out on the reference grid. In Section 4.2 this analysis is complemented by an investigation on the influence of the grid resolution. The numerical results are time-averaged and compared with the experimental LDA data [31]. The LDA measurements are averaged over a long time period of about 1370 dimensionless time units (based on $U_{\infty}$ and $D$ ), whereas the LES results (on the reference grid) are averaged over a shorter period of approximately 150 dimensionless time units. Relying on the investigations of García-Villalba et al. [10] this is sufficient for converged statistics of the first and second-order moments. 
Computers \& Mathematics with Applications, vol. 75 (7), pp. 2338-2355, (2018).

\subsubsection{Time-averaged results in the symmetry $x$-z-plane}

The present study focuses on the detailed analysis of the time-averaged velocity field and the associated time-averaged Reynolds stresses in the symmetry plane (see Figs. 7, 8 and 9) combined with time-averaged wall-streamlines depicted in Fig. 10. This approach permits to clearly identify the previously mentioned characteristic flow regions named according to the investigations of Savory and Toy [21, 22]. Please note that the size and location of these regions in the present results can not be directly compared with those of Savory and Toy, since the inflow conditions (Reynolds number, turbulence intensity and height of the boundary layer) significantly differ. Thus, only the terminology is relevant here.

Figure 7 presents the comparison of the time-averaged first-order moments obtained by the LDA measurements ${ }^{2}$ and the LES predictions with either the injection method STIG-D or STIG-R for the inflow turbulence within the computational domain at $x / D \approx-1.5$. Furthermore, the second-order moments are compared in Fig. 8. Figure 9 shows the profiles of the same quantities at specific locations in the symmetry plane in order to provide a critical quantitative comparison.

First, the oncoming flow upstream of the hemisphere in the region $-1.5 \leq x / D \leq-0.75$ is analyzed. The experimental results show that the thickness of the approaching boundary layer is matching the height of the hemisphere well with $z / D \approx 0.5$ at $x / D=-1.5$. A comparable velocity distribution is visible in both large-eddy simulations. However, some differences in the mean velocity profiles between both methods appear after the injection plane for $x / D \geq-1$ as visible in Fig. 9(a). The results corresponding to the enhanced STIG-R method (red line) are in better agreement with the measurements (black symbols). The discrepancies in the streamwise and wall-normal velocities are even more obvious in the horseshoe vortex area at $x / D \approx-0.6$ (see Figs. 9(a) and 9(b)). The size of the horseshoe vortex system directly depends on the turbulence intensity of the approaching flow [31]: With increasing turbulence intensity the overall size of the vortex system is reduced. Comparing Fig. 8(c) with Fig. 8(b) or the corresponding profiles in Fig. 9(c) highlights the fact that the STIG-R method leads to a slightly closer agreement of the streamwise velocity fluctuations with the LDA data. Consequently, the horseshoe vortex system computed by STIG-R is attenuated and fits better to the experiments (see Figs. 7(d), 7(e) and 7(f)). A rough estimation ${ }^{3}$ of the position of the detachment point yields $x_{\text {detach }}^{\mathrm{EXP}} / D \approx-1.0$ in the measurements. In the simulations values of $x_{\operatorname{detach}}^{\mathrm{STIG}-\mathrm{D}} / D=-1.22$ and $x_{\operatorname{detach}}^{\mathrm{STl}-\mathrm{R}} / D=-0.82$ are predicted. Note that a summary of these characteristics is given in Table 1.

As obvious in Fig. 10 depicting the streamlines close to the surface of the plate and the hemisphere, the entire width of the horseshoe vortex system and of the associated vortical structures in the wake are also reduced by the application of STIG-R in comparison to STIG-D. Here the differences in the results between both injection techniques become clearly visible.

As explained in [31] the horseshoe vortex system in front of the obstacle is composed of pairs of counter-rotating vortices. For STIG-D two pairs of vortices are present, whereas only one vortex pair is visible for STIG-R. The position of the stagnation point is also slightly influenced by the STIG method. With the enhanced STIG-R method it moves upward from $\theta_{\mathrm{stag}}^{\mathrm{STIG}-\mathrm{D}}=161^{\circ}$ to $\theta_{\text {stag }}^{\text {STIG-R }}=163^{\circ}$, where $\theta$ is defined mathematically positive from the back. Thus, the results of the STIG-R injection technique are in closer agreement with the experimental reference

\footnotetext{
${ }^{2}$ Measurements were carried out in the symmetry plane for $-1.5 \leq x / D \leq 2$ and a measuring grid consisting of 2041 measuring points. For further details we refer to [31].

${ }^{3}$ The LDA measuring grid has a streamwise grid spacing of $\Delta x / D=0.05$ above the hemisphere and $\Delta x / D=0.1$ before and after the obstacle allowing to roughly determine the relevant positions.
} 


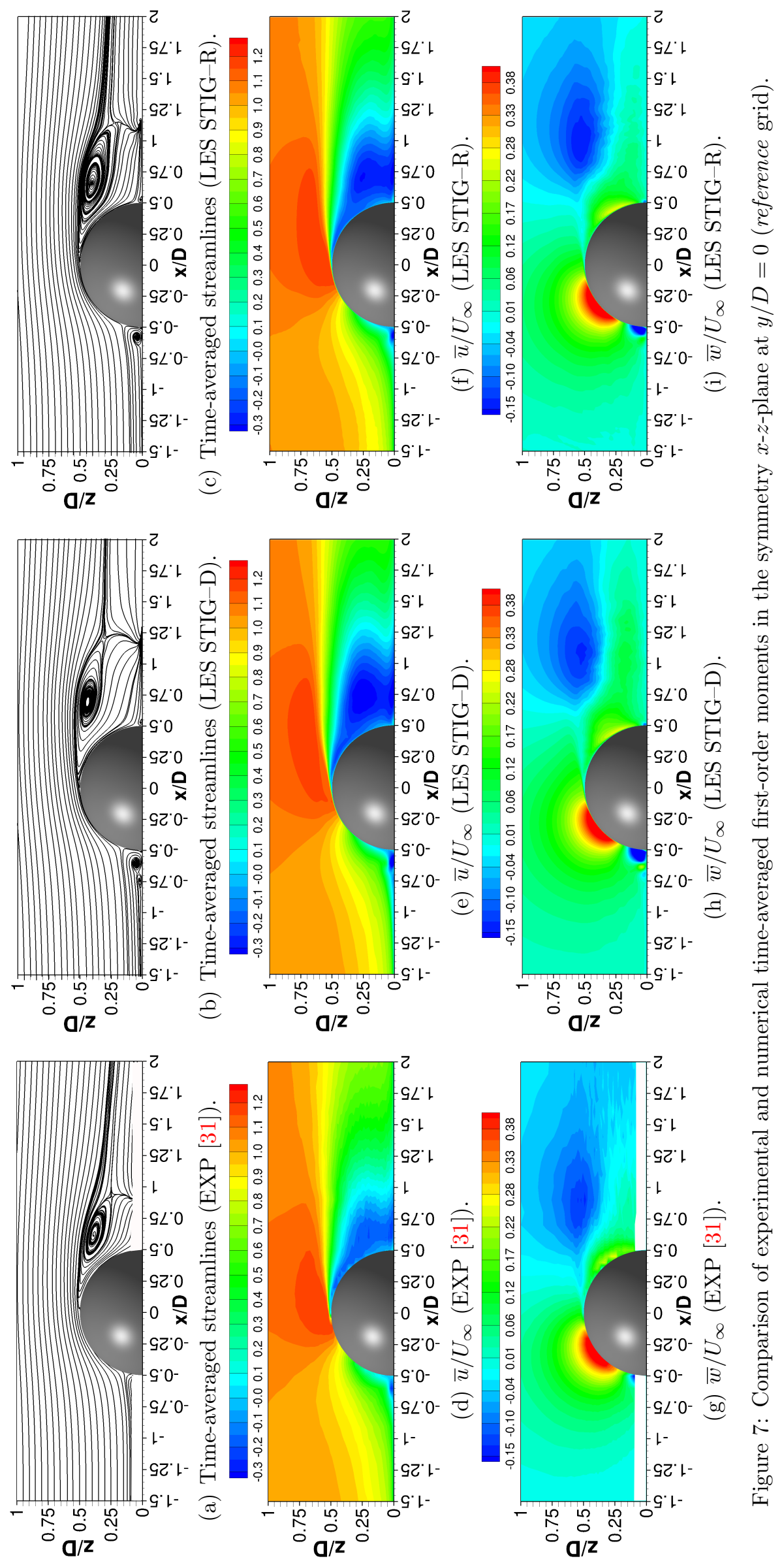



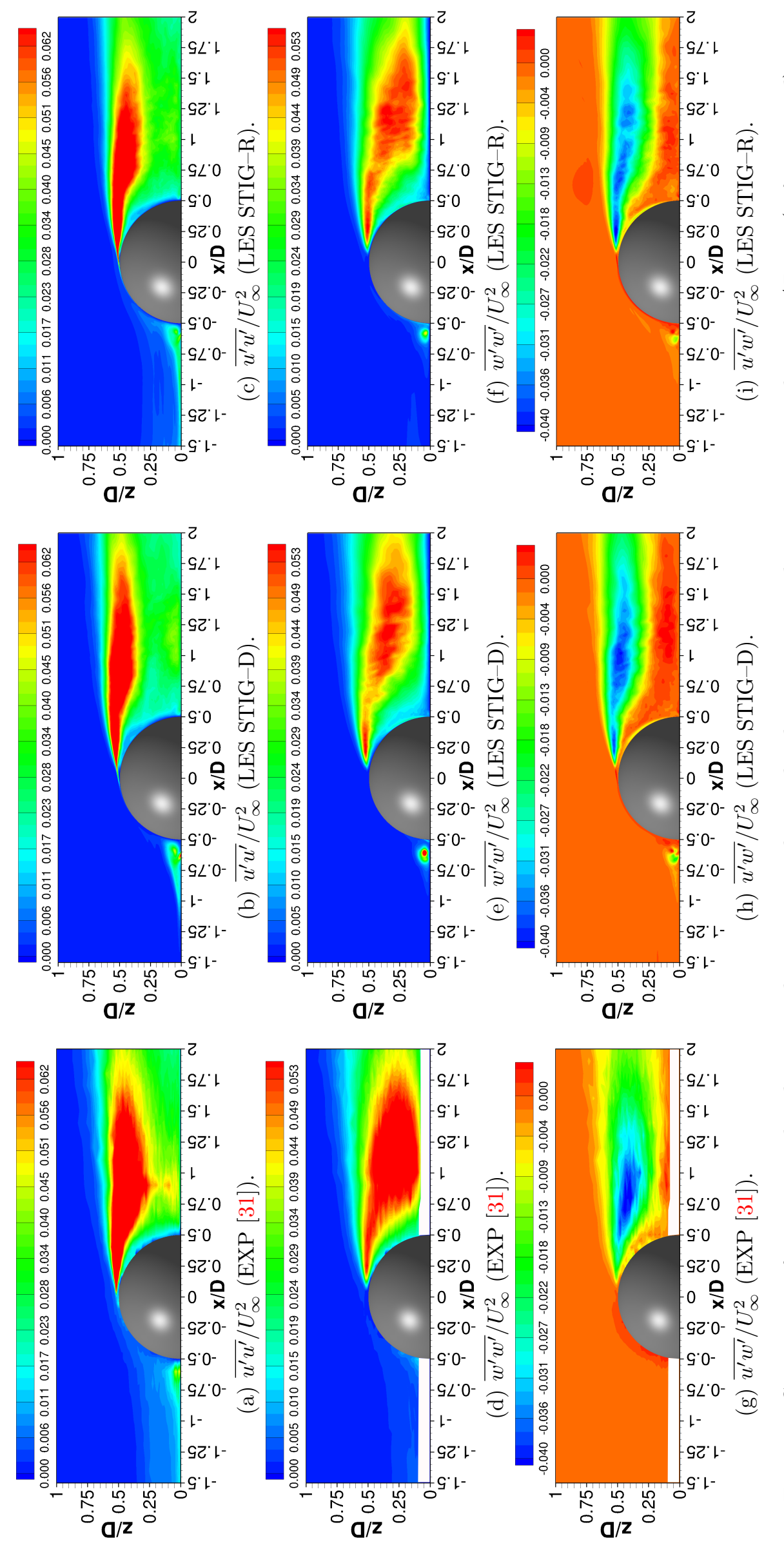


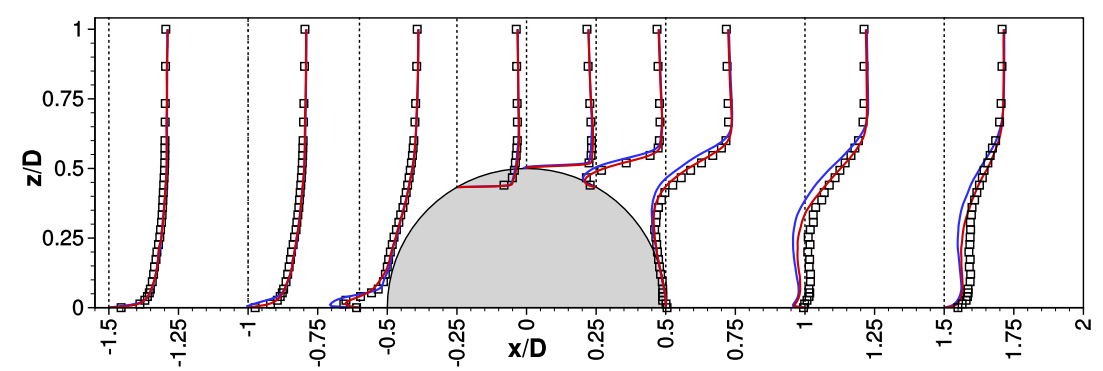

(a) $0.21 \bar{u} / U_{\infty}+x / D$.

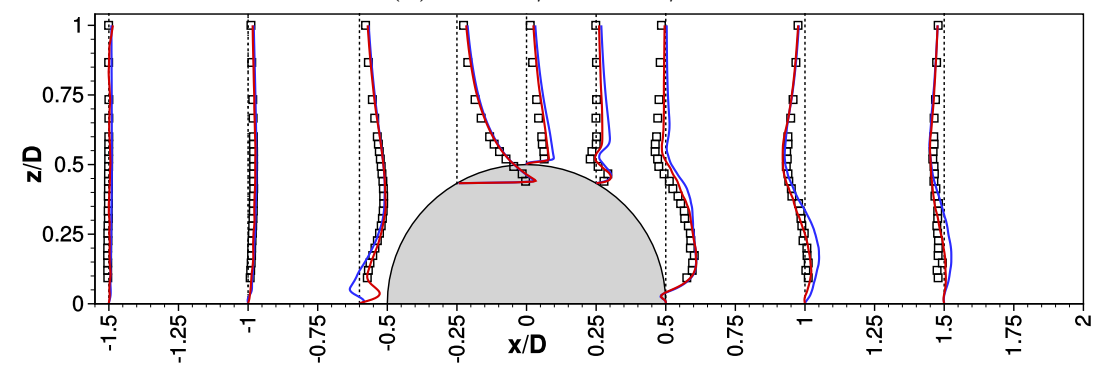

(b) $0.55 \bar{w} / U_{\infty}+x / D$.

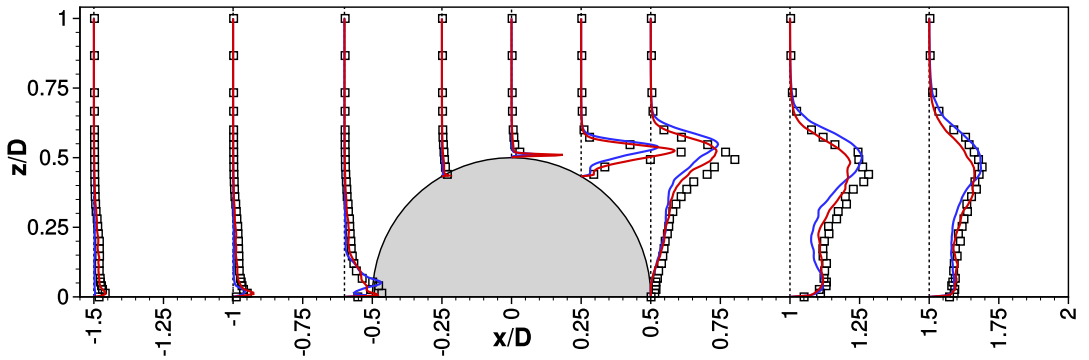

(c) $3 \overline{u^{\prime} u^{\prime}} / U_{\infty}^{2}+x / D$.

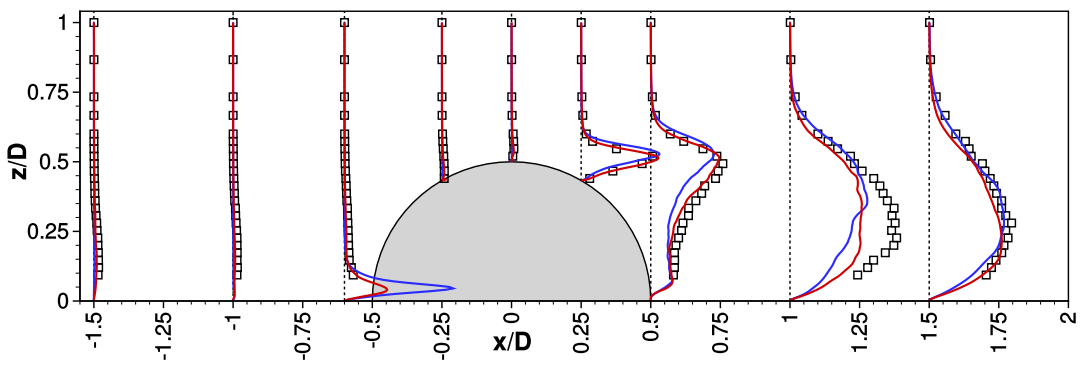

(d) $5 \overline{w^{\prime} w^{\prime}} / U_{\infty}^{2}+x / D$.

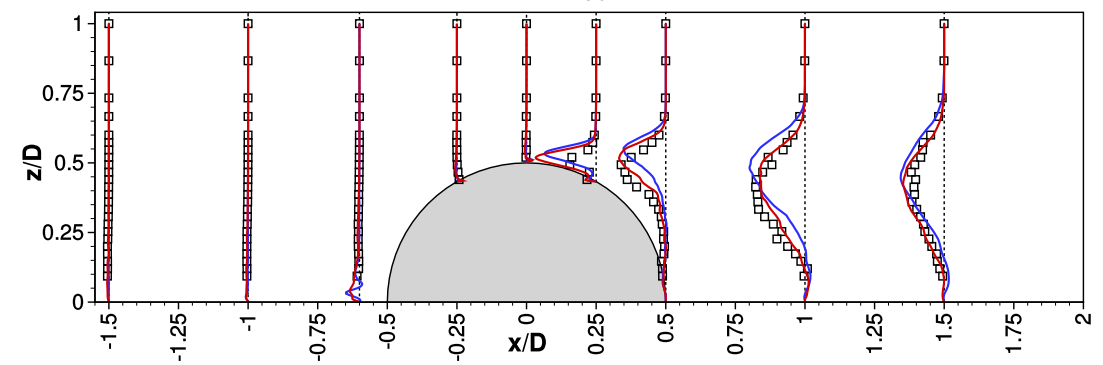

(e) $5 \overline{u^{\prime} w^{\prime}} / U_{\infty}^{2}+x / D$.

Figure 9: Comparison of the experimental (black symbols) and numerical (blue lines: STIG-D, red lines: STIG-R) time-averaged velocities and Reynolds stresses in the symmetry $x$-z-plane at $y / D=0$ and $x / D=$ $\{-1.5,-1,-0.6,-0.25,0,0.25,0.5,1,1.5\}$ (only every second measurement point is displayed) (reference grid). 


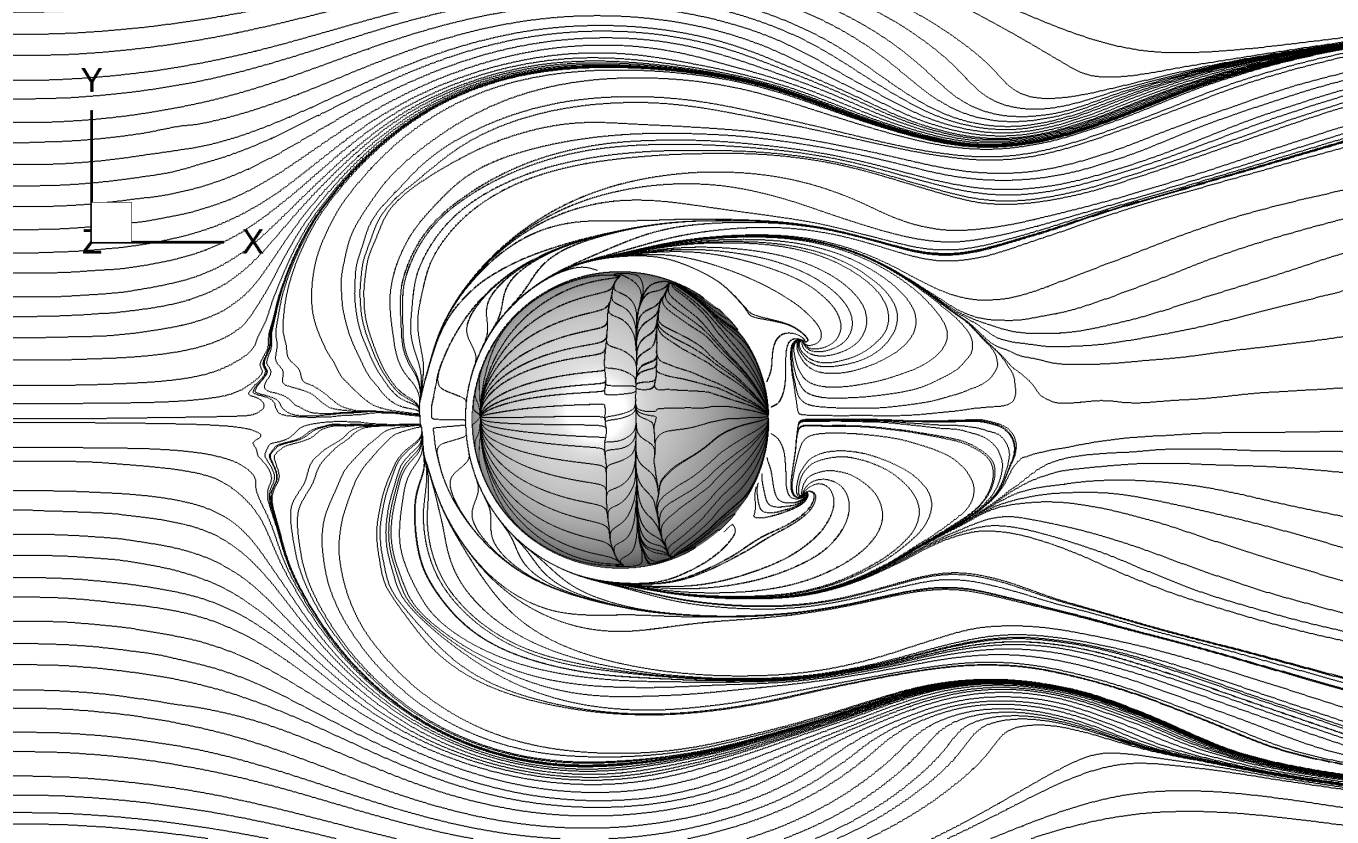

(a) LES STIG-D

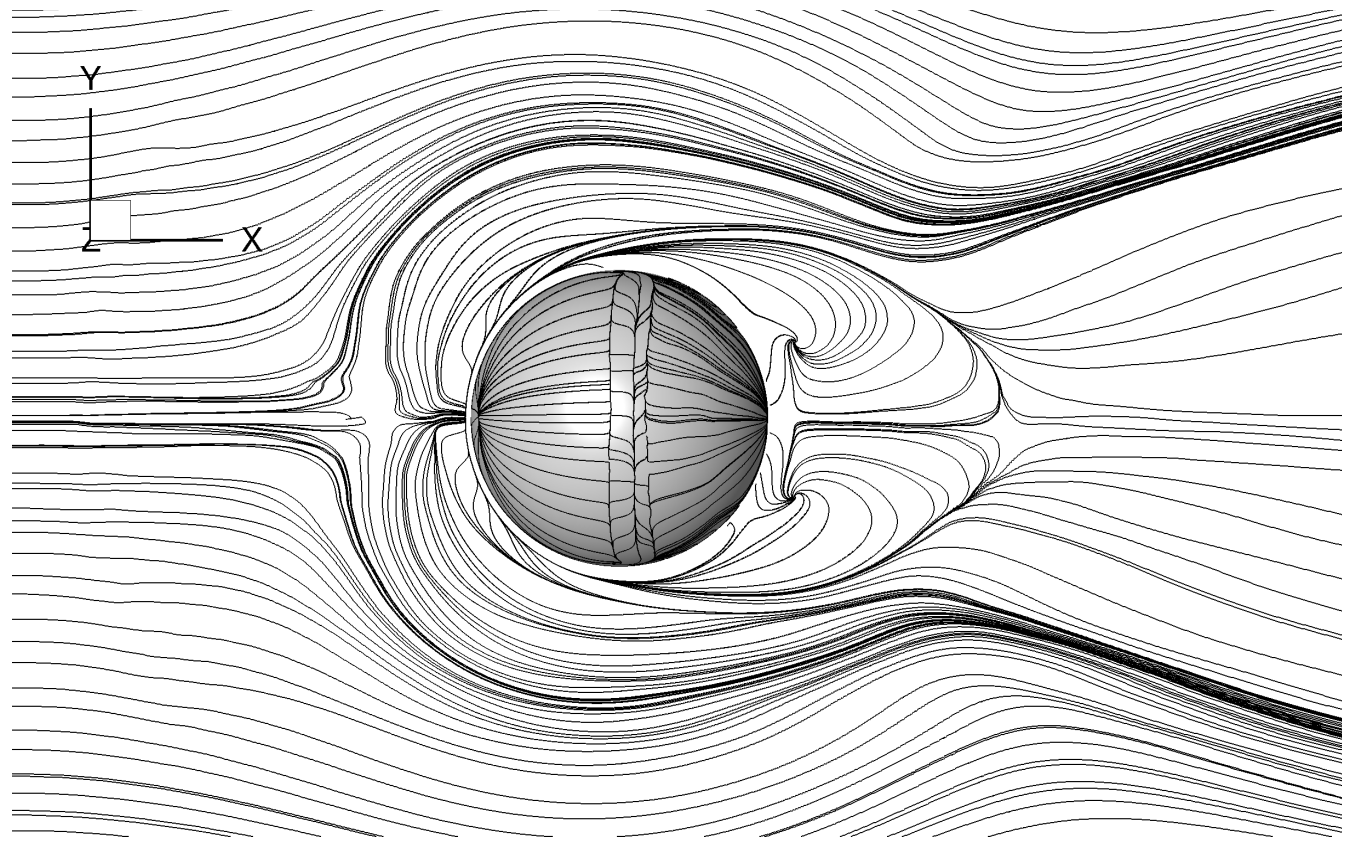

(b) LES STIG-R

Figure 10: Comparison of the time-averaged wall-streamlines near the bottom wall and the surface of the hemisphere (reference grid).

data $\theta_{\text {stag }}^{\mathrm{EXP}} \approx 166^{\circ}$.

For both techniques a good agreement is achieved in the next region to be discussed, i.e., the acceleration area (see Fig. 9). Here, the flow is attached to the wall (see Fig. 10). The incoming turbulence intensities do not play a key role. Thus, no differences are visible between the results obtained by both STIG techniques.

Another interesting location is the separation point on the surface of the hemisphere in the midplane. It marks an important characteristic for the validation of numerical simulations. Its position depends on various influencing parameters (Reynolds number, turbulence intensity of the boundary layer and surface roughness). The predicted flow detaches in the midplane at 
an angle of $\theta_{\text {sep }}^{\text {STIG-D }} \approx 96^{\circ}$ and $\theta_{\text {sep }}^{\text {STIG-R }} \approx 92^{\circ}$ for the LES based on the STIG-D and STIG-R injection methods, respectively. Compared to the LDA measurements $\left(\theta_{\text {sep }}^{\operatorname{EXP}} \approx 90^{\circ}\right)$ the LES with STIG-R produces once more slightly better results.

The separated flow evolves into a free shear layer. This phenomenon can be observed as a strong velocity gradient between the outer flow field and the recirculation area in the wake. For all quantities in this characteristic region $(0 \leq x / D \leq 0.5)$ depicted in Fig. 9 the corresponding results predicted with the STIG-R (red line) are always in closer agreement with the measurements than the data of STIG-D (blue lines). The size of the recirculation area can be evaluated by the reattachment point of the time-averaged flow in the symmetry plane. The measurements roughly give $x_{\text {reattach }}^{\mathrm{EXP}} / D \approx 1.04$. Both LES predict a slightly too long recirculation area with $x_{\text {reattach }}^{\mathrm{STIG}} / D=1.39$ and $x_{\text {reattach }}^{\mathrm{STIG}-\mathrm{R}} / D=1.25$ (see Fig. 10). These minor differences in the length of the recirculation area between experimental and numerical results are in accordance with the observation that the flow separates earlier in the simulations $\left(\Delta \theta=6^{\circ}\right.$ for STIG-D and $2^{\circ}$ for STIG-R). As expected the outcome of STIG-R fits better to the reference data.

The zones of the recirculation area are nearly identical as visible in Fig. 10. However, some differences are noticed in the distributions of the Reynolds stresses (Figs. 8 and 9). The zones of high magnitudes of the normal Reynolds stresses $\overline{u^{\prime} u^{\prime}} / U_{\infty}^{2}$ and the Reynolds shear stresses $\overline{u^{\prime} w^{\prime}} / U_{\infty}^{2}$ are slightly shorter for STIG-R than for STIG-D. Moreover, a significant enhancement of the shape and the amplitude of the wall-normal Reynolds stresses $\overline{w^{\prime} w^{\prime}} / U_{\infty}^{2}$ is observed in Fig. 9(d) for STIG-R in relation to the experimental data. That also holds true for the Reynolds shear stress $\overline{u^{\prime} w^{\prime}} / U_{\infty}^{2}$ depicted in Fig. 9(e). Thus, the overall distribution of the first and second-order moments behind the bluff body are improved by the enhanced injection method STIG-R compared to the STIG-D technique both relying on the identical data generated by the digital filter method.

\subsubsection{Investigation on the synthetic turbulence in the upstream region}

Concluding the discussion in the previous section, it is obvious that the flow predicted by the STIG-R method on the reference grid fits better to the measurements than the one obtained by the STIG-D method on the same grid and with the same physical influence area given by Eq. (11). In order to analyze the reason for this behavior in more detail, an investigation on the distribution of the turbulence quantities just after the STIG injection plane is carried out.

Figure 11 depicts the turbulence level Tu $\mathrm{u}_{\text {inflow }}=\sqrt{\frac{1}{3}\left(\overline{u^{\prime} u^{\prime}}+\overline{v^{\prime} v^{\prime}}+\overline{w^{\prime} w^{\prime}}\right)} / U_{\infty}$ measured by LDA and computed with LES at $x / D=-1.5$. Regarding the distribution of the experimental values of $\mathrm{Tu}_{\text {inflow }}$ some components of the Reynolds stresses could not be measured in the vicinity of the flat plate for $z / D<8 \times 10^{-2}$ and thus are missing. As mentioned before, to fill this gap DNS data from Schlatter et al. [24] are used below this height.

Obviously, the STIG-D method induces a significantly lower level of turbulence (error of about $60 \%$ in the lower part), whereas the STIG-R results agree quite well with the reference data. This significant deviation for STIG-D leads to the already mentioned differences in the incoming flow in front of the hemisphere:

- Slightly stronger turbulent fluctuations $\overline{u^{\prime} u^{\prime}} / U_{\infty}^{2}$ reach the hemispherical body when applying the STIG-R method (compare Figs. 8(b) and 8(c) or the profiles in Fig. 9(c)).

- Based on STIG-R the stronger mixing process leads to a shorter horseshoe vortex system in front of the hemisphere (compare Figs. 7(e) and 7(f)). Only one pair of counterrotating vortices is observed in Fig. 7(c) for this case. As clearly visible from the top 


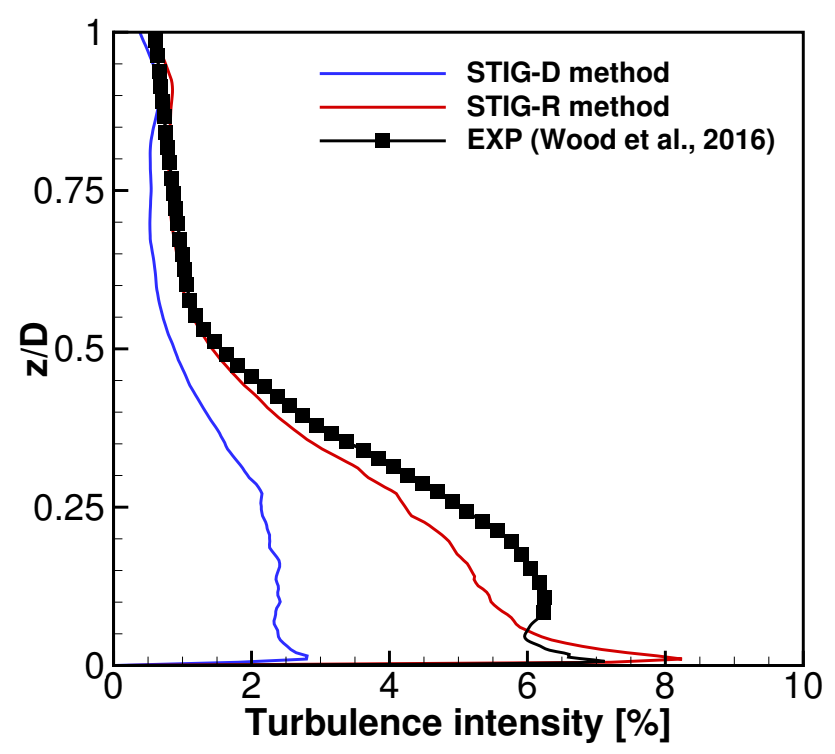

Figure 11: Experimental and numerical time-averaged turbulence level $\mathrm{Tu}_{\text {inflow }}$ at $x / D=-1.5$ (reference grid).

view in Fig. 10 the entire width of the horseshoe vortex system and the associated flow patterns are smaller.

Overall the STIG-R injection method yields a better representation of the flow field in all regions compared to the STIG-D technique. Therefore, the remaining analysis is restricted to the enhanced STIG-R method.

\subsection{Influence of the grid resolution on the injection process}

Two approaches exist to build the 3D influence area as mentioned in Section 2. Based on a grid, which is fine in comparison to the integral length scale, the Gaussian weighting (Eq. (9)) is applied as it was the case for the airfoil configuration presented in Schmidt and Breuer [26]. In the present case the integral length scale is smaller than the cell size (i.e., $L_{\text {inf }}<2 \Delta d_{0}$ ) even on the reference grid with more than 30 million CVs. Therefore, the scaling approach according to Eq. (11) is applied. Since the reference grid delivers results in very close agreement with the measurements, it does not make sense to refine the grid in order to study the influence of the resolution on the outcome. However, a grid coarsening reduces the CPU-time consumption which is of special interest for the planned, even more resource consuming coupled FSI simulations. Therefore, the medium grid described in Section 3.2 is used for this purpose. The grid coarsening has an effect on the injection of the synthetic data: As the cell size increases on each side of the injection plane, the scaling factor $1.3063 L / \Delta d_{0}$ has to be adjusted and leads to $S_{\phi}^{\mathrm{CFD}} \approx 0.33 S_{\phi}^{\text {syn }}$ in the present case. The rest of the computational setup remains the same.

Figure 12 compares the time-averaged streamlines and velocity components in the symmetry $x-z$-plane at $y / D=0$ computed on the medium and the reference grid. Accordingly, Fig. 13 summarizes the time-averaged Reynolds stresses in the symmetry plane computed on both grids. As before profiles of the first and second-order moments at specific locations in the symmetry plane are compared in Fig. 14. The reference results are averaged over a period of approximately 150 dimensionless time units, which is sufficient as explained above. Nevertheless, due to the reduced CPU-time consumption, the results on the medium mesh are averaged 


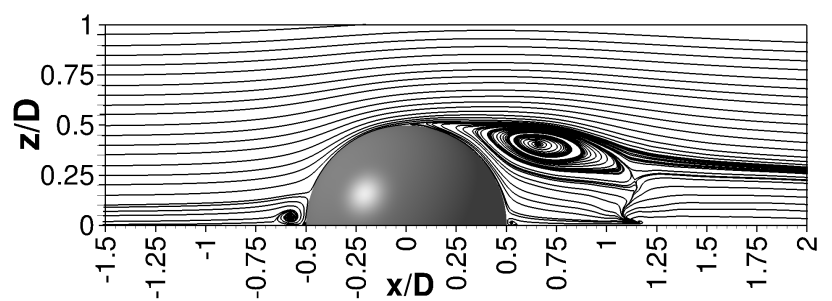

(a) Time-averaged streamlines (reference grid).

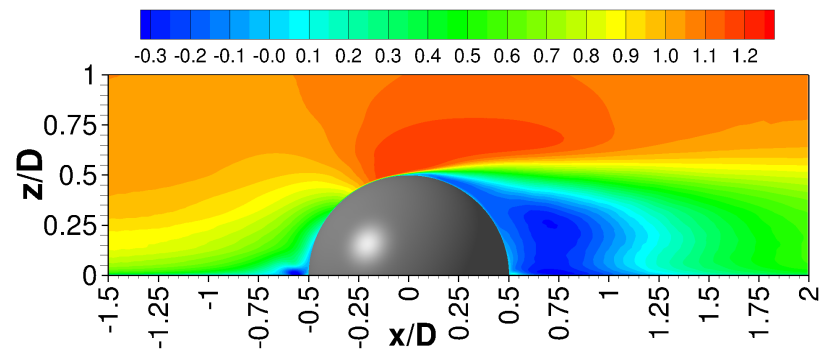

(c) $\bar{u} / U_{\infty}$ (reference grid).

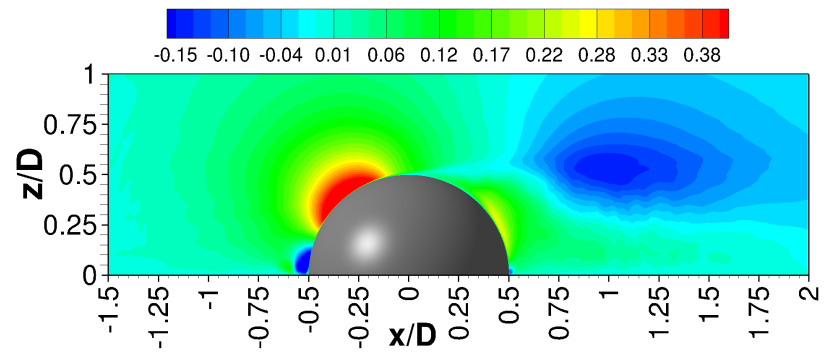

(e) $\bar{w} / U_{\infty}$ (reference grid).

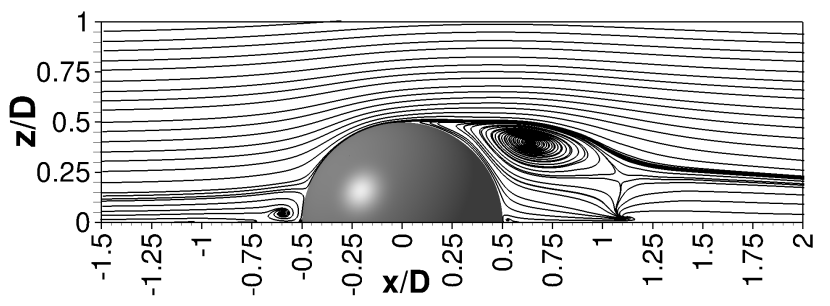

(b) Time-averaged streamlines (medium grid).

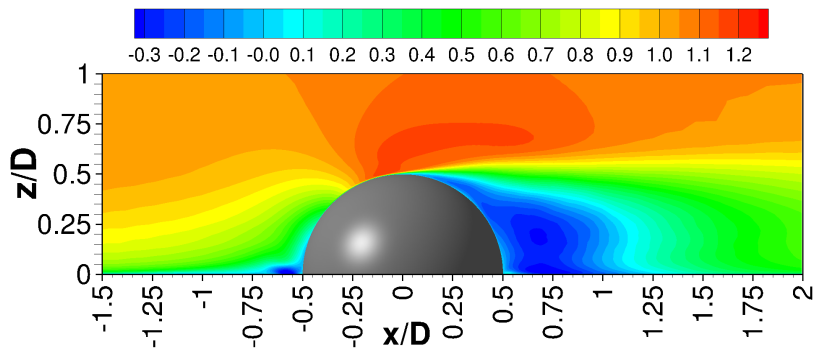

(d) $\bar{u} / U_{\infty}$ (medium grid).

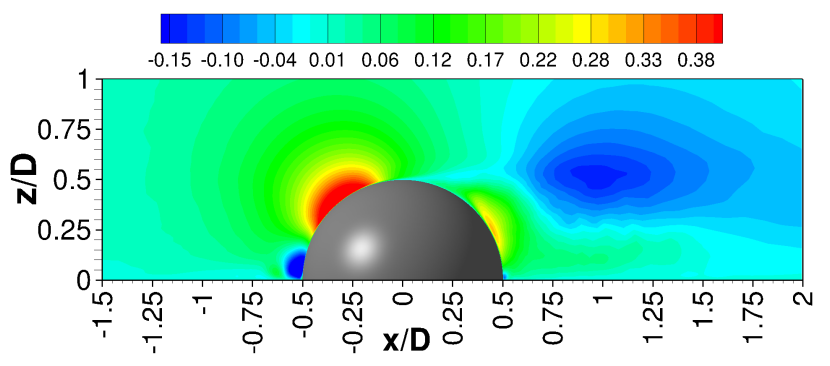

(f) $\bar{w} / U_{\infty}$ (medium grid).

Figure 12: Comparison of the time-averaged streamlines and velocity components in the symmetry $x-z-$ plane at $y / D=0$ predicted by LES on the reference and medium grid applying the STIG-R method.

over a longer time interval (more than 200 dimensionless time units) to provide even smoother statistics.

The different characteristic regions are correctly predicted on the medium grid (see Figs. 12 and 13). Despite some limited discrepancies in the profiles as visible in Fig. 14 the size and location of the horseshoe vortex system remains very similar: The approaching boundary layer detaches from the ground at $x_{\text {detach }}^{\mathrm{STIG}-\mathrm{R} \text { medium }} / D=-0.85$ on the medium grid compared to $x_{\text {detach }}^{\mathrm{STIG}-\mathrm{R} \text { fine }} / D=-0.82$ in the reference case. The stagnation point at the bottom front of the hemisphere is predicted at about $\theta_{\text {stag }}^{\text {STIG-R_medium }}=162^{\circ}$ on the medium grid and at about $\theta_{\text {stag }}^{\text {STIG-R_fine }}=163^{\circ}$ on the fine grid. The recirculation areas are almost identical: The flow detaches at an angle of $\theta_{\text {sep }}^{\text {STIG-R_medium }}=90^{\circ}$ for the simulation on the medium grid and at an angle of $\theta_{\text {sep }}^{\mathrm{STIG}-\mathrm{R} \_ \text {fine }}=92^{\circ}$ on the fine grid. The flow reattaches at about $x_{\text {reattach }}^{\mathrm{STIG} \text {. }}$ case of the medium grid and at about $x_{\text {reattach }}^{\mathrm{STIG}} / D=1.25$ in the reference case. In the vicinity of the hemisphere the time-averaged velocity components and the Reynolds stresses computed on the medium grid are in close agreement with the reference data. Some discrepancies appear for the Reynolds stresses in the wake at $x / D \approx 1$. Nevertheless, except of this region a good agreement of the medium and fine-grid results is achieved regarding the time-averaged velocity components and Reynolds stresses. That clearly demonstrates that the STIG-R injection method combined with the adequate physical influence area leads to results which are in close agreement with the measurements and do not strongly depend on the grid resolution.

Table 1 summarizes the LES results obtained by the two different injection methods and on the 


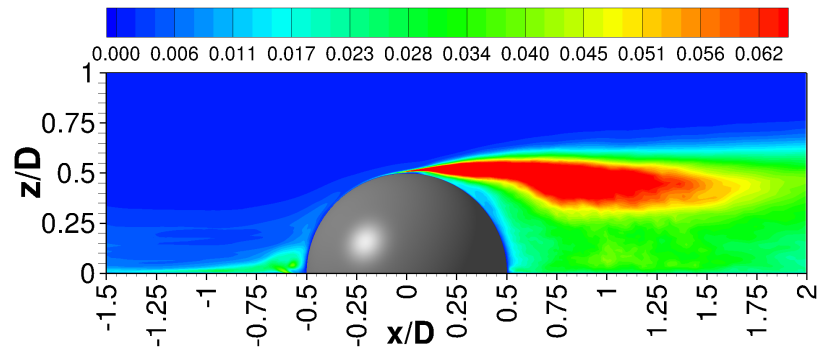

(a) $\overline{u^{\prime} u^{\prime}} / U_{\infty}^{2}$ (reference grid).

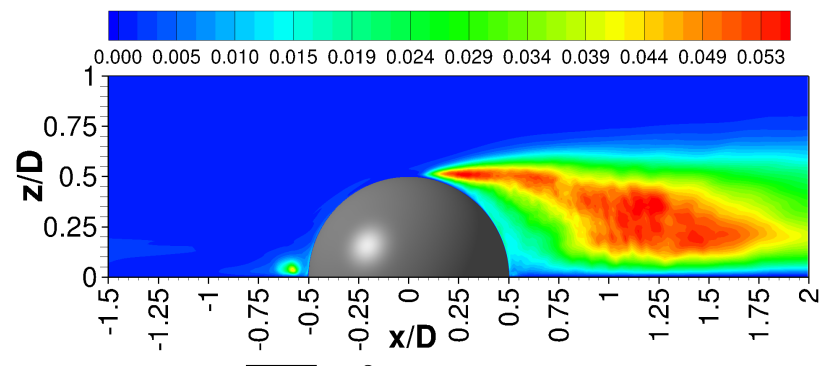

(c) $\overline{w^{\prime} w^{\prime}} / U_{\infty}^{2}$ (reference grid).

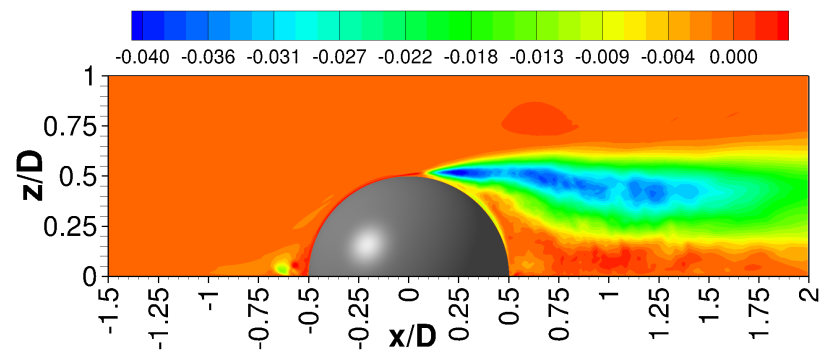

(e) $\overline{u^{\prime} w^{\prime}} / U_{\infty}^{2}$ (reference grid).

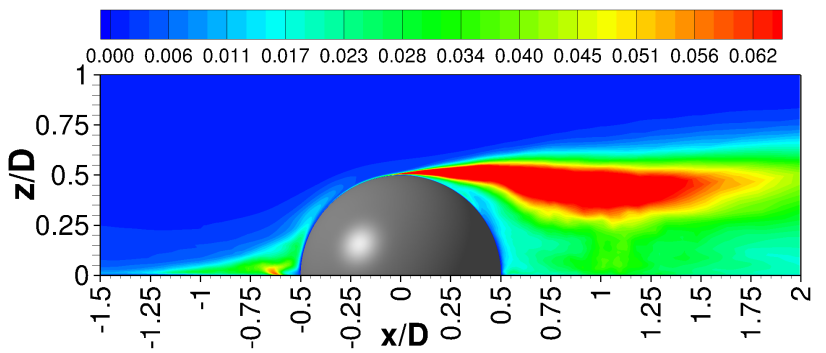

(b) $\overline{u^{\prime} u^{\prime}} / U_{\infty}^{2}$ (medium grid).

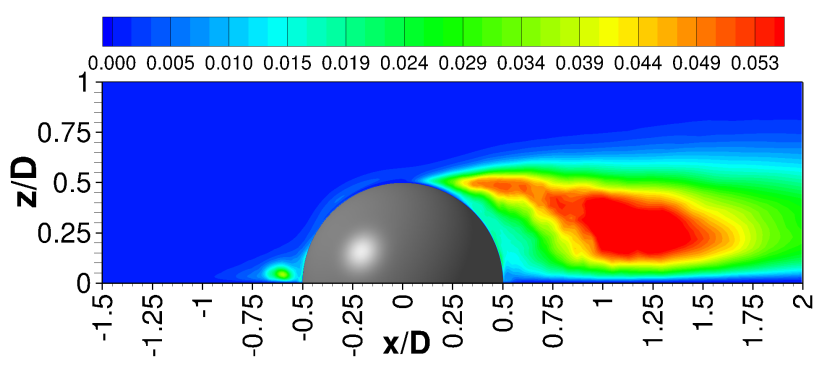

(d) $\overline{w^{\prime} w^{\prime}} / U_{\infty}^{2}$ (medium grid).

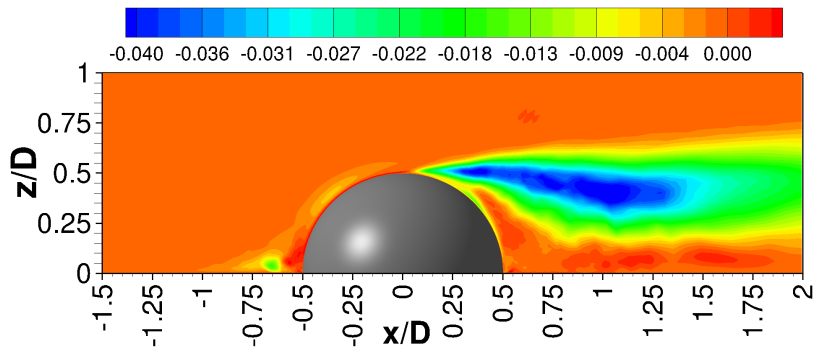

(f) $\overline{u^{\prime} w^{\prime}} / U_{\infty}^{2}$ (medium grid).

Figure 13: Comparison of the time-averaged Reynolds stresses in the symmetry $x-z-$ plane at $y / D=0$ predicted by LES on the reference and medium grid applying the STIG-R method.

Table 1: Flow characteristics predicted by LES on the reference and medium grid and with the STIG-D or STIG-R method compared with the experimental data [31]. All values are determined in the midplane.

\begin{tabular}{llcccl}
\hline \multicolumn{1}{c}{ Flow characteristics } & \multicolumn{4}{c}{ LES } & Exp. [31] \\
& \multicolumn{4}{c}{ reference grid } & medium grid \\
& & STIG-D & STIG-R & STIG-R & \\
\hline Detachment & $x_{\text {detach }} / D$ & -1.22 & -0.82 & -0.85 & $\approx-1.0$ \\
Stagnation & $\theta_{\text {stag }}$ & $161^{\circ}$ & $163^{\circ}$ & $162^{\circ}$ & $\approx 166^{\circ}$ \\
Separation & $\theta_{\text {sep }}$ & $96^{\circ}$ & $92^{\circ}$ & $90^{\circ}$ & $\approx 90^{\circ}$ \\
Reattachment & $x_{\text {reattach }} / D$ & 1.39 & 1.25 & 1.27 & $\approx 1.04$ \\
\hline
\end{tabular}

two grids. In comparison with the experimental data [31] it is obvious that the STIG-R method performs better than STIG-D for the same adequate influence area given by Eq. (11). STIG-R is able to inject synthetic turbulent data correctly even if the integral length scale is smaller than the cell size. This is an important feature, particularly, in case of practical applications with complex geometries and corresponding grid resolutions which are often not optimal in all regions. Furthermore, it has been demonstrated that the predicted results on the medium grid are reliable, since the first and second-order moments are in very good agreement with the reference data. Thus, the STIG-R injection method is not sensitive to the grid resolution. 


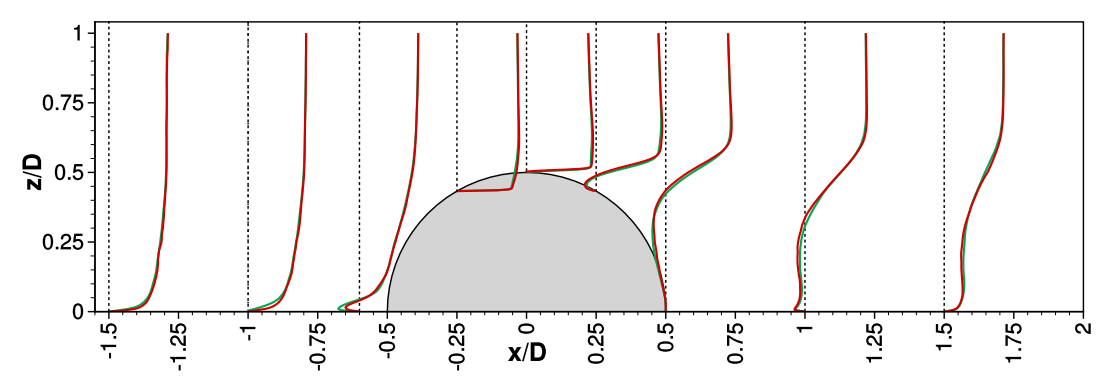

(a) $0.21 \bar{u} / U_{\infty}+x / D$.

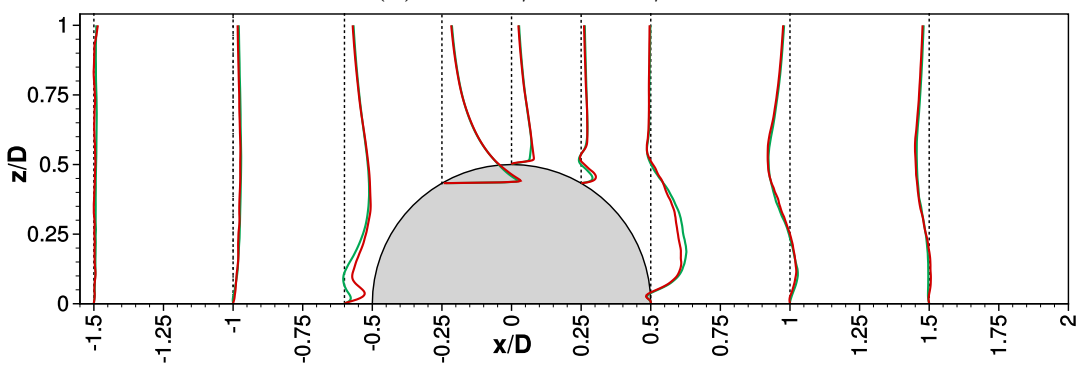

(b) $0.55 \bar{w} / U_{\infty}+x / D$.

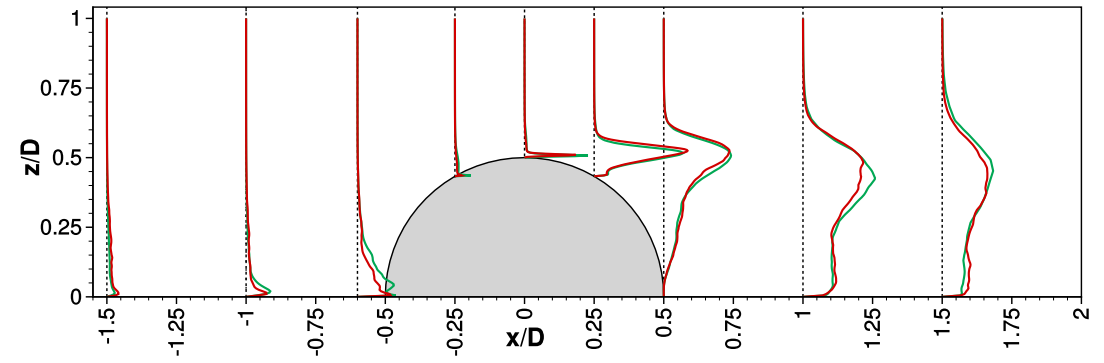

(c) $3 \overline{u^{\prime} u^{\prime}} / U_{\infty}^{2}+x / D$.

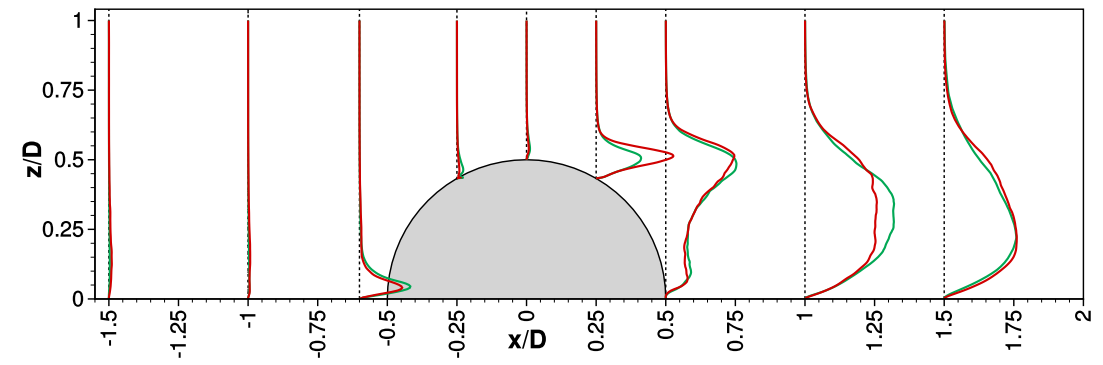

(d) $5 \overline{w^{\prime} w^{\prime}} / U_{\infty}^{2}+x / D$.

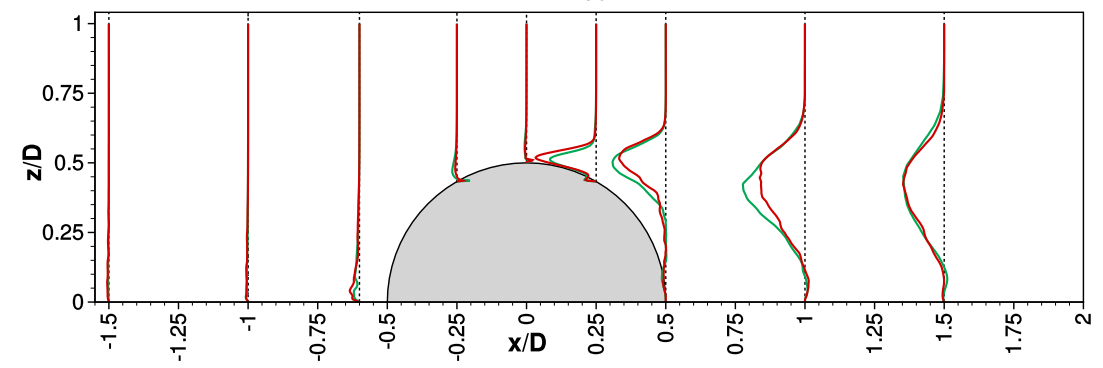

(e) $5 \overline{u^{\prime} w^{\prime}} / U_{\infty}^{2}+x / D$.

Figure 14: Comparison of the time-averaged velocities and Reynolds stresses in the symmetry $x-z-$ plane at $y / D=0$ and $x / D=\{-1.5,-1,-0.6,-0.25,0,0.25,0.5,1,1.5\}$ on the reference (red lines) and medium (green lines) grid applying the STIG-R method. 
Computers \& Mathematics with Applications, vol. 75 (7), pp. 2338-2355, (2018).

\section{CONCLUSIONS}

\section{Conclusions}

In the present study two injection methods based on a source term formulation [27, 26] are evaluated and extended. Their purpose is to mimic realistic inflow conditions for eddy-resolving simulations in case of flow predictions especially for external flows, where typical grids do not allow to prescribe synthetically generated turbulence directly at the inlet of the computational domain due to strong damping effects. Thus, to circumvent this problem the turbulent fluctuations are injected closer to the zone of interest inside the domain. The first formulation (STIG-D) exhibits two drawbacks. It does not preserve the autocorrelations and requires an ad-hoc modification of the 3D influence area in order to get the correct level of turbulence after the injection plane as shown in Wood et al. [31]. The enhanced source term formulation STIG- $\mathrm{R}$ is known to preserve the autocorrelations [26]. However, in the study by Schmidt and Breuer [26] the streamwise integral length scale was larger than the cell size, so that the 3D influence area could be easily defined using a Gaussian weighting function with a width equal to twice the integral length scale. The present work evaluates the enhanced STIG-R formulation combined with a novel extension of the $3 \mathrm{D}$ influence area for the case that the integral length scale in streamwise direction is smaller than the grid resolution. This situation is often encountered in practical applications. The numerical results of both injection methods are analyzed based on the flow around a wall-mounted hemisphere and compared with the experimental data [31].

Both STIG-D and STIG-R techniques combined with a physically motivated 3D influence area predict all characteristic features of the flow. However, the enhanced STIG-R method leads to a better agreement with the experimental data in the entire computational domain. The upstream region is particularly sensitive, since the incoming turbulence has a direct impact on the vortical structures developing in the vicinity of the obstacle. STIG-R represents the incoming flow more accurately. A slightly shorter horseshoe vortex system is induced. Only one pair of counter-rotating vortices is observed contrary to two pairs of vortices for the case with STIG-D. The application of STIG-R also leads to improvements in the separation and reattachment regions. The flow detaches from the hemisphere further downstream leading to a size of the recirculation area fitting better to the experimental data. The main reason for these deviations is the significantly low level of turbulence induced by STIG-D in the region close to the injection plane. Therefore, the enhanced injection technique STIG-R is considered clearly superior to STIG-D.

To evaluate the performance of STIG-R combined with the physically determined 3D influence area for a broader application range, an investigation based on a coarsened grid is carried out. For this medium grid the integral length scale is considerably smaller than the cell size of the injection plane. Consequently, the source terms have to be redefined according to the suggested formulation taking the integral length scale into account. The resulting time-averaged first and second-order moments show a very good agreement with the reference data illustrating on the one hand the potential of the present enhanced injection method STIG-R and on the other hand its insensitivity with respect to the grid resolution.

Finally, it has to be noted that the injection method is not restricted to the digital filter concept but can be combined with other techniques to generate (synthetic) turbulent inflow data. Furthermore, it is not restricted to large-eddy simulations but can be applied in all kinds of eddy-resolving methods. 


\section{Acknowledgments}

The numerical part of the project is financially supported by the Deutsche Forschungsgemeinschaft (BR 1847/12-2). The computations were carried out on SuperMUC at LRZ Munich (pr84na, pr53ne). Furthermore, the authors thank M. Klein (Universität der Bundeswehr München) for providing the original source code of the digital filter based inflow procedure.

\section{References}

[1] Abel, M., Stojkovic, D., Breuer, M., 2006. Nonlinear stochastic estimation of wall models for LES. International Journal of Heat and Fluid Flow 27 (2), 267-278.

[2] Adrian, R. J., 2007. Hairpin vortex organization in wall turbulence. Physics of Fluids 19 (4), 041301.

[3] Bailly, C., Comte-Bellot, G., 2015. Turbulence. Springer.

[4] Breuer, M., 2007. Boundary conditions for LES. In: Wagner, C., Hüttl, T., Sagaut, P. (Eds.), Chap. 5.2 of Large-Eddy Simulation for Acoustics. Cambridge University Press.

[5] Breuer, M., De Nayer, G., Münsch, M., Gallinger, T., Wüchner, R., 2012. Fluid-structure interaction using a partitioned semi-implicit predictor-corrector coupling scheme for the application of large-eddy simulation. Journal of Fluids and Structures 29, 107-130.

[6] Breuer, M., Kniazev, B., Abel, M., 2007. Development of wall models for LES of separated flows using statistical evaluations. Computers \& Fluids 36 (5), 817-837.

[7] Breuer, M., Peller, N., Rapp, C., Manhart, M., 2008. Flow over periodic hills - numerical and experimental study in a wide range of Reynolds numbers. Computers \& Fluids 38 (2), 433-457.

[8] Ferziger, J. H., Perić, M., 2002. Computational Methods for Fluid Dynamics, 3rd Edition. Springer Berlin.

[9] Fröhlich, J., von Terzi, D. A., 2008. Hybrid LES/RANS methods for the simulation of turbulent flows. Progress in Aerospace Sciences 44 (5), 349-377.

[10] García-Villalba, M., Li, N., Rodi, W., Leschziner, M. A., 2009. Large-eddy simulation of separated flow over a three-dimensional axisymmetric hill. Journal of Fluid Mechanics $627,55-96$.

[11] Germano, M., Piomelli, U., Moin, P., Cabot, W. H., 1991. A dynamic subgrid-scale eddy viscosity model. Physics of Fluids A 3, 1760-1765.

[12] Keating, A., Piomelli, U., Balaras, E., Kaltenbach, H.-J., 2004. A priori and a posteriori tests of inflow conditions for large-eddy simulation. Physics of Fluids 16, 4696-4712.

[13] Kempf, A., Wysocki, S., Pettit, M., 2012. An efficient, parallel low-storage implementation of Klein's turbulence generator for LES and DNS. Computers \& Fluids 60, 58-60.

[14] Klein, M., Sadiki, A., Janicka, J., 2003. A digital filter based generation of inflow data for spatially-developing direct numerical or large-eddy simulations. Journal of Computational Physics 186, 652-665. 
[15] Lesieur, M., 2008. Turbulence in Fluids, 4th Edition. Springer.

[16] Lund, T. S., Wu, X., Squires, K. D., 1998. Generation of turbulent inflow data for spatially-developing boundary layer simulations. Journal of Computational Physics 140, $223-258$.

[17] Moser, R. D., Kim, J., Mansour, N. N., 1999. Direct numerical simulation of channel flow up to $R e_{\tau}=590$. Physics of Fluids 11 (4), 943-945.

[18] Nicoud, F., Ducros, F., 1999. Subgrid-scale stress modelling based on the square of the velocity gradient tensor. Flow, Turbulence and Combustion 62 (3), 183-200.

[19] Piomelli, U., Chasnov, J. R., 1996. Large-eddy simulations: Theory and applications. In: Hallbäck, M., Henningson, D., Johansson, A., Alfredson, P. (Eds.), Turbulence and Transition Modeling. Kluwer, pp. 269-331.

[20] Sagaut, P., Deck, S., Terracol, M., 2013. Multiscale and multiresolution approaches in turbulence - LES, DES and hybrid RANS/LES methods: Applications and guidelines (second edition). Imperial College Press.

[21] Savory, E., Toy, N., 1986. The flow regime in the turbulent near wake of a hemisphere. Experiments in Fluids 4, 181-188.

[22] Savory, E., Toy, N., 1986. Hemisphere and hemisphere-cylinders in turbulent boundary layers. Journal of Wind Engineering and Industrial Aerodynamics 23, 345-364.

[23] Scheit, C., Nusser, K., Hager, G., Becker, S., Zeiser, T., Wellein, G., 2014. Optimizing the FASTEST-3D CFD code for massive parallelism. In: 26th Int. Conf. on Comp. Fluid Dynamics, ParCFD 2014. Norway, Trondheim.

[24] Schlatter, P., Orlu, R., Li, Q., Brethouwer, G., Fransson, J. H. M., Johansson, A. V., Alfredsson, P. H., Henningson, D. S., 2009. Turbulent boundary layers up to $\operatorname{Re}_{\theta}=2500$ studied through simulation and experiment. Physics of Fluids 21 (5), 51702.

[25] Schmidt, S., Breuer, M., 2015. Extended synthetic turbulence inflow generator within a hybrid LES-URANS methodology for the prediction of non-equilibrium wall-bounded flows. Flow, Turbulence and Combustion 95 (4), 669-707.

[26] Schmidt, S., Breuer, M., 2017. Source term based synthetic turbulence inflow generator for eddy-resolving predictions of an airfoil flow including a laminar separation bubble. Computers \& Fluids 146, 1-22.

[27] Schmidt, S., Breuer, M., 2018. Application and extension of a synthetic turbulence inflow generator within a hybrid LES-URANS methodology. In: Grigoriadis, D., Geurts, B., Kuerten, H., Fröhlich, J., Armenio, V. (Eds.), ERCOFTAC Series, Direct and Large-Eddy Simulation X, 10th Int. ERCOFTAC Workshop on Direct and Large-Eddy Simulation: DLES-10, Limassol, Cyprus, May 27-29, 2015. Vol. 24. Springer Int. Publishing AG, pp. 63-69.

[28] Smagorinsky, J., 1963. General circulation experiments with the primitive equations I: The basic experiment. Monthly Weather Review 91 (3), 99-165. 
[29] Spille-Kohoff, A., Kaltenbach, H. J., 2001. Generation of turbulent inflow data with a described shear-stress profile. In: Liu, C., Sakell, L., Beutner, T. (Eds.), DNS/LES Progress and Challenges, Proc. of the Third AFOSR Int. Conf. on DNS/LES, Arlington, USA. Greyden Press: Columbus, pp. 319-326.

[30] Tabor, G., Baba-Ahmadi, M., 2010. Inlet conditions for large-eddy simulation: A review. Computers \& Fluids 39, 553-567.

[31] Wood, J. N., De Nayer, G., Schmidt, S., Breuer, M., 2016. Experimental investigation and large-eddy simulation of the turbulent flow past a smooth and rigid hemisphere. Flow, Turbulence and Combustion 97 (1), 79-119. 